Tôhoku Math. Journ. 30 (1978), 177-210.

\title{
ON A GENERALIZATION OF THE HOPF FIBRATION, II*
}

(Complex structures on the products of generalized Brieskorn manifolds)

KinetSU ABE

(Received February 12, 1976)

This paper is the continuation of our previous paper under the same title [2], which we refer to as Part I. In Part I, we studied contact structures on the generalized Brieskorn manifolds as a generalization of the connection form of the Hopf fibration. Our objective in this paper is to study complex structures on the products of generalized Brieskorn manifolds. These complex structures may be considered as a generalization of the Calabi-Eckmann complex structures [6]. Therefore, we are particularly interested in studying those aspects of the complex manifolds which Calabi and Eckmann studied on the products of odd dimensional spheres. In fact, we show that our complex manifolds possess properties analogous to the Calabi-Eckmann manifolds.

Let $\Sigma_{1}$ and $\Sigma_{2}$ be generalized Brieskorn manifolds; see Part I for the definition and examples. First we show that $\Sigma_{1} \times \Sigma_{2}$ admits a complex structure which is intimately related to the normal contact structures on $\Sigma_{1}$ and $\Sigma_{2}$. It is then shown that this complex structure admits no Kählerian structure and that $\Sigma_{1} \times \Sigma_{2}$ is the total space of a holomorphic fibration on a complex analytic space $B_{1} \times B_{2}$. This fibration, unlike the Calabi-Eckmann case, is not necessarily a fiber bundle; however, the fibers are elliptic curves. Our first main result is that any analytic subvariety of $\Sigma_{1} \times \Sigma_{2}$ has the induced fibration over a complex analytic space. In particular, the fibers are the only irreducible analytic subvarieties of dimension 1; hence, they have no singular point. Earlier, Calabi and Eckmann have shown that their complex manifolds possess the same property [6]. However, their proof does not apply to our case directly, for $\Sigma_{1} \times \Sigma_{2}$ may have non-vanishing middle homology and $B_{1} \times B_{2}$ may not admit a projective imbedding.

Next, we show that many of the $\Sigma_{1} \times \Sigma_{2}$ 's admit infinitely many seemingly different complex structures. Indeed, many such examples are constructed. After introducing a criterion to distinguish these complex

* Research partially supported by NSF Grant MPS 74-07184 A01.

This paper consists of part of the author's doctoral thesis submitted to Tohoku University. 
structures, we show that $\Sigma_{1} \times \Sigma_{2}$ admits infinitely many distinct complex structures if either $\Sigma_{1}$ or $\Sigma_{2}$ is a Brieskorn sphere (exotic or standard) or a generalized lens space. It is also shown that $S^{1} \times S^{2} \times \cdots \times S^{4 k-1}$ admits infinitely many distinct complex structures.

Some observations are made concerning the automorphisms of $\Sigma_{1} \times \Sigma_{2}$. For instance, we show that the set of infinitesimal automorphisms of $\Sigma_{1} \times \Sigma_{2}$ which keep the fiber fixed is isomorphic to $\boldsymbol{C}$ (over $\boldsymbol{C}$ ). We also mention the vanishing of the Chern numbers on $\Sigma_{1} \times \Sigma_{2}$.

In concluding this introduction, we point out that the above classification of complex structures is still crude, and we hope that a more precise classification can be made in the near future. It also seems quite reasonable that some kind of classification can be made by means of deformation. Fortunately, the fundamental machinery of deformation is available. In fact, Morita [20] has recently given a classification of complex structures on $S^{1} \times \Sigma$ in terms of the homotopy of the underlying almost complex structures, where $\Sigma$ is a Brieskorn sphere.

Finally, we thank J. Erbacher for our discussions with him during his stay at Connecticut. We also thank Professor S. Sasaki for reading this manuscript.

Complex structures on products of generalized Brieskorn manifolds. In the proof of Theorem 2 in Part I, we showed that the contact structure on a generalized Brieskorn manifold $\Sigma$ is normal, i.e., the torsion tensor of that contact structure $[X, Y]+\phi[\phi X, Y]+\phi[X, \phi Y]-[\phi X, \phi Y]-$ $(X \eta(Y)-Y \eta(X)) \xi=0$ everywhere, where $\phi$ is the $(1,1)$ tensor of the associated almost contact Riemannian structure. Making use of this fact, we show the following theorem.

TheOREM 1. Let $\Sigma_{1}$ and $\Sigma_{2}$ be two generalized Brieskorn manifolds. Then $\Sigma_{1} \times \Sigma_{2}$ admits a complex structure, and also $\Sigma_{1} \times S^{1}$ admits $a$ complex structure.

Proof. Here we assume that $\Sigma_{1}$ and $\Sigma_{2}$ are the intersection of given varieties and the unit sphere. We do not lose any generality by assuming so by Theorem 3 in Part I. It is well known by a theorem of NewlanderNirenberg [22] that an almost complex structure $J$ on a smooth manifold is a complex structure if and only if its torsion $T J(X, Y)=[X, Y]+$ $J[J X, Y]+J[X, J Y]-[J X, J Y]=0$. Now let $\eta_{1}$ and $\eta_{2}$ be the normal contact structures on $\Sigma_{1}$ and $\Sigma_{2}$, respectively. Let $\left(\phi_{1}, \xi_{1}, \eta_{1}\right)$ and $\left(\phi_{2}, \xi_{2}, \eta_{2}\right)$ be the associated almost contact structures on $\Sigma_{1}$ and $\Sigma_{2}$, respectively. Let $T \Sigma_{1}$ and $T \Sigma_{2}$ be the tangent bundles of $\Sigma_{1}$ and $\Sigma_{2}$, respectively. Then the tangent bundle $T\left(\Sigma_{1} \times \Sigma_{2}\right)$ of $\Sigma_{1} \times \Sigma_{2}$ can be naturally represented 
by the exterior direct sum of $T \Sigma_{1}$ and $T \Sigma_{2}$, which we will denote by $T \Sigma_{1} \hat{\oplus} T \Sigma_{2}$. Then any tangent vector $X$ of $\Sigma_{1} \times \Sigma_{2}$ can be written uniquely as $X=X_{1} \hat{\oplus} X_{2}$ where $X_{1}$ and $X_{2}$ are the $T \Sigma_{1}$ and $T \Sigma_{2}$-components of $X$, respectively. Now making use of $\phi_{1}$ and $\phi_{2}$, we define an almost complex structure $J$ on $\Sigma_{1} \times \Sigma_{2}$ as follows.

$$
J X=\left(\dot{\phi}_{1} X_{1}-\eta_{2}\left(X_{2}\right) \xi_{1}\right) \hat{\oplus}\left(\phi_{2} X_{2}+\eta_{1}\left(X_{1}\right) \xi_{2}\right) .
$$

Then, we have

$$
\begin{aligned}
J^{2} X= & J\left\{\left(\phi_{1} X_{1}-\eta_{2}\left(X_{2}\right) \xi_{1}\right) \hat{\oplus}\left(\dot{\phi}_{2} X_{2}+\eta_{1}\left(X_{1}\right) \xi_{2}\right\}\right. \\
= & \left\{\phi_{1}\left(\phi_{1} X_{1}-\eta_{2}\left(X_{2}\right) \xi_{1}\right)-\eta_{2}\left(\phi_{2} X_{2}+\eta_{1}\left(X_{1}\right) \xi_{2}\right) \xi_{1}\right\} \\
& \hat{\oplus}\left\{\phi_{2}\left(\phi_{2} X_{2}+\eta_{1}\left(X_{1}\right) \xi_{2}\right)+\eta_{1}\left(\phi_{1} X_{1}-\eta_{2}\left(X_{2}\right) \xi_{1}\right) \xi_{2}\right\} \\
= & \left\{\phi_{1}^{2} X_{1}-\eta_{1}\left(X_{1}\right) \xi_{1}\right\} \hat{\oplus}\left\{\phi_{2}^{2} X_{2}-\eta_{2}\left(X_{2}\right) \xi_{2}\right\} \\
= & \left(-X_{1}\right) \hat{\oplus}\left(-X_{2}\right)=-\left(X_{1} \hat{\oplus} X_{2}\right)=-X .
\end{aligned}
$$

Hence, $J^{2}$ is an almost complex structure.

The rest of the proof follows from the argument of Morimoto [19]. q.e.d.

The theorem follows immediately from Theorem 2 in Part I and a result of Morimoto [19]. Nevertheless, for the sake of later use, let us describe how a complex structure is obtained from the normal (almost) contact structure discussed in Part I along the line of Morimoto's arguments.

As was pointed out in Part I, Brieskorn [4] showed that an odd dimensional homotopy sphere bounding a parallelizable manifold has infinitely many Brieskorn manifold representations. Thus, we have

CoRollary 1. Let $\Sigma_{1}$ and $\Sigma_{2}$ be two Brieskorn spheres (exotic or standard). Then $\Sigma_{1} \times \Sigma_{2}$ admits infinitely many seemingly different complex structures.

REMarK 1. As Brieskorn and van de Ven [5] pointed out, by a result of $\mathrm{R}$. de Sapio, products of two exotic spheres are usually diffeomorphic to products of two standard spheres except for the case that one of the factors is $S^{1}$ and the other is an exotic sphere.

CoROLlary 2. a) $S^{n} \times S^{n+1} \times S^{m} \times S^{m+1}(n, m \geqq 2)$ admits infinitely many seemingly different complex structures, where $n$ and $m$ are even.

b) $S^{1} \times S^{2} \times \cdots \times S^{4 k-1}$ admits infinitely many seemingly different complex structures.

Proof. a) follows from the result of Kauffman [15] as is mentioned 
in Corollary 2 in Part I. As for b), $S^{2} \times S^{3} \times S^{4} \times \cdots \times S^{4 k-1}$ can be decomposed into the product of $\left(S^{2} \times S^{3}\right),\left(S^{4} \times S^{5}\right), \cdots,\left(S^{4 k-2} \times S^{4 k-1}\right)$, each of which has representations as Brieskorn manifolds by [15]. Remove one of those products, say $S^{i} \times S^{i+1}$, and combine it with $S^{1}$, and match up the rest of the products into pairs. Then by Theorem 1, each of these pairs admits infinitely many seemingly different complex structures; and therefore, the products of these pairs admit infinitely many seemingly different complex structures in a natural way.

CoRollary 3. Let $L\left(p, q_{1}, \cdots, q_{n-1}\right)$ and $L\left(p^{\prime}, q_{1}^{\prime}, \cdots, q_{n-1}^{\prime}\right)$ be two generalized lens spaces. Then $L\left(p, q_{1}, \cdots, q_{n-1}\right) \times L\left(p^{\prime}, q_{1}^{\prime}, \cdots, q_{n-1}^{\prime}\right)$ admits a complex structure.

Proof. This follows from Corollary 5 in Part I and Theorem 1.

q.e.d.

These complex structures are closely related to those of Hopf [13], Calabi-Eckmann, and Brieskorn-van de Ven [5]. In fact, by mimicing the method of Brieskorn-van de Ven [5], we can construct more complex structures on $\Sigma_{1} \times \Sigma_{2}$ as follows: Define an action $f_{\tau}$ of $\boldsymbol{C}$ on $\left(V_{1}\right)_{0} \times\left(V_{2}\right)_{0}$ by

$$
\begin{aligned}
f_{\tau}(t ; & \left.Z_{0}, \cdots, Z_{m} ; \omega_{0}, \cdots, \omega_{n}\right) \\
& =\left(e^{2 \pi p_{0} t} Z_{0}, \cdots, e^{2 \pi p_{m} t} Z_{m} ; e^{2 \pi q_{0} t \tau} \omega_{0}, \cdots, e^{2 \pi q_{n} t \tau} \omega_{n}\right) .
\end{aligned}
$$

Here $\tau$ is a complex number such that $\operatorname{Im} \tau \neq 0,\left(p_{0}, \cdots, p_{m}\right)$ and $\left(q_{0}, \cdots, q_{n}\right)$ are positive integers. Then one can construct a diffeomorphism of $\boldsymbol{C} \times$ $\Sigma_{1} \times \Sigma_{2}$ onto $\left(V_{1}\right)_{0} \times\left(V_{2}\right)_{0}$ by defining $(t, z, \omega) \mapsto f_{\tau}(t ; z, \omega)$. It can be seen that the action $f_{\tau}$ is a free, holomorphic, locally proper action; therefore, by [12], the quotient space, say $H\left(p_{0}, \cdots, p_{m} ; q_{0}, \cdots, q_{n}\right)$ is a complex manifold. Our above diffeomorphism, then, induces a diffeomorphism from $\Sigma_{1} \times \Sigma_{2}$ onto $H\left(p_{0}, \cdots, p_{m} ; q_{0}, \cdots, q_{n}\right)$. Therefore, $\Sigma_{1} \times \Sigma_{2}$ admits a complex structure.

Let $S^{2 p+1}$ and $S^{2 q+1}$ be two odd dimensional standard spheres, and let these spheres admit the normal contact structures which are induced from the Hopf fibrations. Then it is, of course, well known that $S^{2 p+1} \times$ $S^{2 q+1}$ admits a complex structure which is naturally induced from these normal contact structures. It is also evident that this complex structure arises among the Calabi-Eckmann structures on $S^{2 p+1} \times S^{2 q+1}$ as a very special but typical example, which carries a lot of information. In a way, our complex structures on $\Sigma_{1} \times \Sigma_{2}$ occupy the corresponding status among the complex structures which are given by mimicking the Brieskornvan de Ven method; however, our complex structures make it possible 
to investigates the interrelations between them and contact structures on the factors. On the other hand, such interrelations between the generalized Brieskorn-van de Ven complex structures and some differential geometric structures on each factor space remain to be investigated. In this sense, it seems to us that our structures are more accessible from the differential geometric point of view.

The following is an immediate generalization of a theorem of CalabiEckmann [6].

THeOREM 2. Let $\Sigma_{1}$ and $\Sigma_{2}$ be two generalized Brieskorn manifolds. Then a) If $\Sigma_{1}$ is simply connected, then the complex structure on $\Sigma_{1} \times S^{1}$ does not admit a Kählerian structure; therefore, $\Sigma_{1} \times S^{1}$ is not projective algebraic.

b) If $\Sigma_{1}$ and $\Sigma_{2}$ are 2-connected, then the complex structure on $\Sigma_{1}$ and $\Sigma_{2}$ does not admit a Kählerian structure; therefore, $\Sigma_{1} \times \Sigma_{2}$ is not projective algebraic. In particular, most of the generalized Brieskorn manifolds in Examples 1, 2 and 3 given in Part I satisfy the above conditions.

PRoof. It is well known that if $M$ is a compact Kählerian manifold, then every odd Betti number is even, and every even Betti number is positive.

Let $M_{1}$ and $M_{2}$ be two compact manifolds, and let $B_{p}\left(M_{1}\right)$ and $B_{q}\left(M_{2}\right)$ be the $p$-th and $q$-th Betti numbers of $M_{1}$ and $M_{2}$, respectively. Then $B_{r}\left(M_{1} \times M_{2}\right)=\sum_{p+q=r} B_{p}\left(M_{1}\right) \times B_{q}\left(M_{2}\right)$. For a), by Hurewicz isomorphism theorem we have $B_{1}\left(\Sigma_{1}\right)=0$. Therefore $B_{1}\left(\Sigma_{1} \times S^{1}\right)=B_{0}\left(\Sigma_{1}\right) \times B_{1}\left(S^{1}\right)+$ $B_{1}\left(\Sigma_{1}\right) \times B_{0}\left(S^{1}\right)=1 \times 1=1$. Thus, $\Sigma_{1} \times S^{1}$ cannot be Kählerian. For b), $B_{2}\left(\Sigma_{1} \times \Sigma_{2}\right)=B_{0}\left(\Sigma_{1}\right) \times B_{2}\left(\Sigma_{2}\right)+B_{1}\left(\Sigma_{1}\right) \times B_{1}\left(\Sigma_{2}\right)+B_{2}\left(\Sigma_{1}\right) \times B_{0}\left(\Sigma_{2}\right)$. Since $\Sigma_{1}$ and $\Sigma_{2}$ are 2-connected, $B_{1}\left(\Sigma_{1}\right)=B_{1}\left(\Sigma_{2}\right)=B_{2}\left(\Sigma_{1}\right)=B_{2}\left(\Sigma_{2}\right)=0$ by Hurewicz isomorphism theorem; therefore, $B_{2}\left(\Sigma_{1} \times \Sigma_{2}\right)=0$. In order to show that they are not projective algebraic, it suffices to point out that any complex submanifold of complex projective space is automatically Kählerian with its induced metric from the Fubini-Study metric. As for the last remark in Theorem 2, it has been shown that Examples 1, 2 and 3 in Part I give highly connected manifolds; for the details, see [4], [23], [24]. q.e.d.

Concerning the proof of Theorem 2, it is known that there are many 3-dimensional manifolds which are associated with Brieskorn polynomials and weighted homogeneous polynomials of three complex variables, and which are not simply connected. In fact, by the work of Mumford [21] a Brieskorn manifold $\Sigma$ associated with a polynomial $P(Z)=Z_{0}^{a_{0}}+Z_{1}^{a_{1}}+Z_{2}^{a_{2}}$ 
is simply connected if and only if the origin is not a singular point. This means that one of $a_{0}, a_{1}$ and $a_{2}$ must be 1 . Consequently, $\Sigma$ must be diffeomorphic to $S^{3}$. Now let $\Sigma$ be any three dimensional Brieskorn manifold whose fundamental group is abelian and contains a copy of $Z$, then $B_{1}(\Sigma)=$ even $>0$. By Poincaré duality, $B_{2}(\Sigma)=B_{1}(\Sigma)=$ even $>0$. Thus $\Sigma \times S^{1}$ or $\Sigma_{1} \times \Sigma_{2}$ (where $\Sigma_{1}$ and $\Sigma_{2}$ satisfy the same conditions as above) satisfies the above necessary conditions to be Kählerian. Therefore, it is still possible that these manifolds admit a Kählerian structure. Also it is clear that the same argument as given to show a) and b) works when $\Sigma_{1}$ and $\Sigma_{2}$ have finite fundamental groups.

Next let us assume that the $(n+1)$-tuple $\left(q_{0}, \cdots, q_{n}\right)$ defining the $C$-action on the variety $V$ consists of all rational numbers. As we mentioned previously, this action induces a complex torus $T=C / \Gamma$ action on $H=V_{\mathrm{o}} / \Gamma$, where $\Gamma$ is the discrete subgroup of $C$ generated by 1 and $\sqrt{-1} d$. We also mentioned that the quotient space $H / T$, say $B$, is a normal complex space such that the natural quotient mapping is holomorphic in the sense of complex (or analytic) space [7], [12]. The orbit space of $\Sigma$ with respect to the $S^{1}$-action coincides with $B$, i.e., $\Sigma / S^{1}=$ $B=H / T$. Let $\Sigma_{i}, B_{i}$ and $\pi_{i}(i=1,2)$ be a generalized Brieskorn manifold, its base space and the quotient map $\pi_{i}: \Sigma_{i} \rightarrow B_{i}$, respectively. Denote by $\pi=\pi_{1} \times \pi_{2}$ the Cartesian product of $\pi_{1}$ and $\pi_{2}$, i.e., $\pi: \Sigma_{1} \times \Sigma_{2} \rightarrow B_{1} \times B_{2}$ is defined by $\pi(x, y)=\left(\pi_{1}(x), \pi_{2}(y)\right)$ for any $(x, y) \in \Sigma_{1} \times \Sigma_{2}$. It is obvious that $\pi$ is a continuous mapping. We will show that $\pi$ is indeed a holomorphic mapping in the sense of complex (or analytic) space under the following condition which we think superfluous. Let $V_{i}(i=1,2)$ be the irreducible varieties defining $\Sigma_{i}(i=1,2)$ as before. Let us denote by $H_{j}(j=0, \cdots, n)$ the complex hyper linear subspace of $C^{n+1}$ given by $H_{j}=\left\{\left(Z_{0}, \cdots, Z_{n}\right) \in C^{n+1}: Z_{j}=0\right\}$. We say that $V_{i}$ is in general position if $V_{i} \cap H_{j}(i=1,2, j=0, \cdots, n)$ is a complete intersection with the origin as its only singular point. Note here that $V_{i} \cap H_{j}-$ the origin $\}$ is a complex submanifold of $H_{j}$ of dimension $k_{i}-1$, where $k_{i}$ is the complex dimension of $V_{i}$. As a matter of remark, we would like to point out that the class of varieties in general position is quite broad and most of the interesting examples lie in this class. For instance, all the varieties of Example 1 in Part I are in general position, and most of the varieties in Examples 2 and 3 in Part $I$ are also in general position. Finally, we say $\Sigma_{i}$ is in general position if it is obtained from the variety $V_{i}$ in general position. Then we have

Lemma 1. Let $\left(\Sigma_{i}, \pi_{i}, B_{i}\right)(i=1,2)$ be fibrations such that $\Sigma_{i}(i=1,2)$ are in general position. Then the mapping $\pi=\pi_{1} \times \pi_{2}: \Sigma_{1} \times \Sigma_{2} \rightarrow B_{1} \times B_{2}$ 
is holomorphic in the sense of complex space. Here $B_{1} \times B_{2}$ has the complex space structure of the Cartesian product of $B_{1}$ and $B_{2}$.

Proof. First of all we point out that $\pi^{-1}(\bar{x}, \bar{y})$ is diffeomorphic to a torus for all $(\bar{x}, \bar{y}) \in B_{1} \times B_{2}$. This can be seen as follows. $\pi_{1}^{-1}(\bar{x})$ is the $S^{1}$-orbit on $\Sigma_{1}$ through a point $x$ in $\pi_{1}^{-1}(\bar{x})$ and $\pi_{2}^{-1}(\bar{y})$ is the $S^{1}$-orbit on $\Sigma_{2}$ through a point $y$ in $\pi_{2}^{-1}(\bar{y})$. These orbits are diffeomorphic to $S^{1}$. Thus $\pi^{-1}(\bar{x}, \bar{y})=\left(\pi_{1}^{-1}(\bar{x}), \pi_{2}^{-1}(\bar{y})\right)=\pi_{1}^{-1}(\bar{x}) \times \pi_{2}^{-1}(\bar{y})$ is diffeomorphic to $S^{1} \times S^{1}$ which is nothing but a torus. By the definition of the complex structure on $\Sigma_{1} \times \Sigma_{2}$, the tangent bundle of $\pi^{-1}(\bar{x}, \bar{y})$ is invariant under the almost complex structure $J$; therefore, $\pi^{-1}(\bar{x}, \bar{y})$ is a complex submanifold of complex dimension 1. Thus $\pi^{-1}(\bar{x}, \bar{y})$ is an elliptic curve and will be called a fiber of $\Sigma_{1} \times \Sigma_{2}$ over $(\bar{x}, \bar{y})$. Next we show that the union of all fibers over pairs $(\bar{x}, \bar{y})$ such that one of $\bar{x}$ and $\bar{y}$ is singular in the previous sense is contained in a union of a finite number of thin subsets of $\Sigma_{1} \times \Sigma_{2}$. Denote this union by $W$. For the definition of thin sets, see Gunning-Rossi [11]. Recall here that we say $\bar{x}$ (or $\bar{y}$ ) is singular in $B_{1}$ (or $B_{2}$ ) if $\pi_{1}^{-1}(\bar{x})$ (or $\pi_{2}^{-1}(\bar{y})$ ) is a singular orbit. Now let $V$ be a variety in general position, and let $t\left(Z_{0}, \cdots, Z_{n}\right)=\left(e^{2 \pi q_{0} t i} Z_{0}, \cdots, e^{2 \pi q_{n} t i} Z_{n}\right), t \in[0, d)$ be the induced $S^{1}-$ action on $V$, where $d$ is the least common multiple of the denominators when $q_{i}(0 \leqq i \leqq n)$ is expressed as a fraction of relatively prime positive integers. As we know, the $S^{1}$-action on $\Sigma$ is the restriction of the $S^{1}$ action on $C^{n+1}$. Thus any point $Z=\left(Z_{0}, \cdots, Z_{n}\right)$ which belongs to $\Sigma$ and to a principal orbit of the $S^{1}$-action on $C^{n+1}$ must belong to a principal orbit of the $S^{1}$-action on $\Sigma$. Since the $S^{1}$-action on $C^{n+1}$ is effective, a point $Z=\left(Z_{0}, \cdots, Z_{n}\right) \in C^{n+1}$ belongs to a singular orbit only if at least one of $Z_{i}$ vanishes. For more details, see Neumann [23] or Jänich [14]. $V \cap H_{j}(j=0, \cdots, n)$ is a subvariety of $H_{j}$ and it is invariant under the $C$-action on $C^{n+1}$. Since $V$ is in general position, $V \cap H_{j}-\{0\}$ is a complex submanifold of $H_{j}$ of complex dimension $k-1$, where $k$ is the complex dimension of $V$. Let $S$ be the unit sphere in $C^{n+1}$ and let $S_{j}$ be the intersection $S_{j}=S \cap H_{j}(j=0, \cdots, n)$. Note that $S_{j}$ is the unit sphere of $H_{j}(j=0, \cdots, n)$. Then applying the same argument as in Lemma 1 in Part I, $S \cap\left(V \cap H_{j}\right)=S_{j} \cap\left(V \cap H_{j}\right)$ is a generalized Brieskorn manifold of dimension $2 k-3$. In fact, this manifold may not be a connected manifold. To be more precise, one should apply the argument of Lemma 1 to each connected component of $V \cap H_{j}-\{0\}(j=0, \cdots, n)$ (or each irreducible branch of $\left.V \cap H_{j}\right)$. Thus $S \cap\left(V \cap H_{j}\right)$ is a disjoint union of a finite number of generalized Brieskorn manifolds. It is clear that $S \cap$ $\left(V \cap H_{j}\right)$ is a submanifold of $\Sigma$ and each connected component admits a normal contact structure as in Theorem 2 in Part I. Also it is not hard 
to see that the associated almost contact Riemannian structures on these connected components are exactly the restriction of that on $\Sigma$ to these components considered as naturally imbedded submanifolds of $\Sigma$. Consequently, applying the argument in Theorem 1 to each component, we see that the Cartesian product of $S \cap\left(V \cap H_{j}\right)$ and any generalized Brieskorn manifold admits a complex structure as given in Theorem 1. It is evident that this Cartesian product is a complex submanifold of the Cartesian product of $\Sigma$ and the above generalized Brieskorn manifold with the complex structure given in Theorem 1. Now denote $W_{1, j}=$ $S_{j} \cap\left(V_{1} \cap H_{j}\right)(j=0, \cdots, m)$ and $W_{2, l}=S_{l} \cap\left(V_{2} \cap H_{l}\right)(l=0, \cdots, n)$, where $m$ and $n$ are the complex dimensions of the ambient complex Euclidean spaces containing $V_{1}$ and $V_{2}$, respectively. Then by the above observation, it is clear that $W$ is contained in $\left(\bigcup_{j=1}^{m}\left(W_{1, j} \times \Sigma_{2}\right)\right) \cup\left(\bigcup_{l=1}^{n}\left(\Sigma_{1} \times W_{2, l}\right)\right)$. Denote this set by $\widetilde{W}$. Since each $W_{1, j} \times \Sigma_{2}$ and $\Sigma_{1} \times W_{2, l}$ are a finite union of complex submanifolds of $\Sigma_{1} \times \Sigma_{2}$ of complex codimension $\leqq 1$, and since a complex submanifold is a thin set, we have shown that $W$ is contained in a union of a finite number of thin sets. Next we show that $\pi=\pi_{1} \times \pi_{2}$ is holomorphic in the sense of complex space. Let $(\bar{x}, \bar{y}) \in$ $B_{1} \times B_{2}$ be a point of $B_{1} \times B_{2}$ such that neither of them is singular. Then by the same argument as given in the proof of Lemma 12 in Part I, there are neighborhoods $U_{1}$ and $U_{2}$ of $\bar{x}$ and $\bar{y}$, respectively, such that the complex structure of $B_{i}$ restricted to $U_{i}(i=1,2)$ is non-singular. In other words, $U_{i}(i=1,2)$ is a complex manifold with respect to the quotient complex structure. Since $B_{1} \times B_{2}$ has the Cartesian product complex structure of $B_{1}$ and $B_{2}$, the neighborhood $U=U_{1} \times U_{2}$ of $(\bar{x}, \bar{y})$ is a complex manifold with respect to the complex structure of $B_{1} \times B_{2}$. It is easy to see that $\pi$ restricted to $\pi^{-1}(U)$ is a smooth mapping and that the Jacobian mapping $\pi_{*}$ of $\pi$ in $\pi^{-1}(U)$ commutes with the complex structure on $\pi^{-1}(U) \subset \Sigma_{1} \times \Sigma_{2}$ and the complex structure on $U$. This can be seen by recalling the definition of $\pi$ and the argument in Lemma 1. Thus $\pi \mid \pi^{-1}(U)$ is holomorphic. Since $(\bar{x}, \bar{y})$ is taken arbitrarily in such a way that neither of them is singular, we have shown that $\pi$ is holomorphic outside $\widetilde{W}$. Now let $(\bar{x}, \bar{y})$ be a point in $\pi(\widetilde{W})$, and let $U$ be an open neighborhood of $(\bar{x}, \bar{y})$. Let $f: U \rightarrow C$ be a continuous function which represents a local section of the structure sheaf of $B_{1} \times B_{2}$ over $U$. Now we want to show that $f \circ \pi$ in $\pi^{-1}(U)$ represents a local section of the structure sheaf of $\Sigma_{1} \times \Sigma_{2}$. In other words, $f \circ \pi$ in $\pi^{-1}(U)$ is a holomorphic function in the usual sense. Clearly $f \circ \pi$ is continuous in $\pi^{-1}(U)$ and it is holomorphic in $\pi^{-1}(U)-\widetilde{W}$ since we showed that $\pi$ in $\Sigma_{1} \times \Sigma_{2}-\widetilde{W}$ is holomorphic. $\widetilde{W}$ being a finite union of thin sets 
implies that $\widetilde{W} \cap \pi^{-1}(U)$ is again a union of a finite number of thin sets in $\pi^{-1}(U)$. Since $f \circ \pi$ is continuous, it is locally bounded. Thus applying the Riemann removable singularity theorem [11], we see that $f \circ \pi$ is holomorphic in $\pi^{-1}(U)$. Thus $\pi$ is holomorphic in the sense of complex (analytic) space.

q.e.d.

Now we have

THEOREM 3. Let $\Sigma_{1}$ and $\Sigma_{2}$ be two generalized Brieskorn manifolds and assume that they are in general position. The triple $\left(\Sigma_{1} \times \Sigma_{2}, \pi\right.$, $B_{1} \times B_{2}$ ) is an analytic fibration with elliptic curves as its fibers. Here $\pi$ is a holomorphic mapping in the sense of complex space. This fibration is in general not a fiber bundle.

Proof. Obvious by virtue of Theorem 1 and Lemma 1. $\left(\Sigma_{1} \times \Sigma_{2}\right.$, $\pi, B_{1} \times B_{1}$ ) becomes a fiber bundle with a torus as its typical fiber if and only if both $\left(\Sigma_{1}, \pi_{1}, B_{1}\right)$ and $\left(\Sigma_{2}, \pi_{2}, B_{2}\right)$ are $S^{1}$-bundles.

q.e.d.

Note here that this last theorem is also an extension of a result of Calabi-Eckmann [6]. They have also shown the following. Let $S^{2 p+1}$ and $S^{2 q+1}$ be two odd dimensional spheres and let $C P^{p}$ and $C P^{q}$ be complex projective spaces of complex dimension $p$ and $q$, respectively. We denote by $S^{2 p+1} \times S^{2 q+1} \stackrel{\pi}{\rightarrow} C P^{p} \times C P^{q}$ the Calabi-Eckmann fibration. Their result states every compact complex subvariety is fibered by tori. The following result is the analogue to their theorem; however, their method cannot be applied directly to our case since $\Sigma_{1}$ and $\Sigma_{2}$ can have non-vanishing middle homologies.

THEOREM 4. Let $\Sigma_{1} \times \Sigma_{2} \stackrel{\pi}{\rightarrow} B_{1} \times B_{2}$ be such an analytic fibration as given in Theorem 3. Let $W$ be a compact complex subvariety of $\Sigma_{1} \times \Sigma_{2}$. Then $W$ is the total space of analytic fibration with tori as its fibers, and the fibration is induced from that of $\Sigma_{1} \times \Sigma_{2}$.

Proof. Without loss of generality, we can assume that $W$ is irreducible. So from now on $W$ is assumed to be irreducible. It is well known that any such subvariety is a cycle in the sense of homology, and denote the cycle by $[W]$ and call it the carrying cycle of $W$. Since $W$ is irreducible, the set of all regular (or simple) points of $W$ is connected, open and dense in $W$. Denote it by $R(W)$. It is also well known that $R(W)$ is a complex manifold of certain pure dimension $k$. In this case $[W]$ is an element of $H^{2 k}\left(\Sigma_{1} \times \Sigma_{2} ; \boldsymbol{R}\right)$ via de Rham theorem, where $H^{2 k}\left(\Sigma_{1} \times \Sigma_{2} ; \boldsymbol{R}\right)$ is the $2 k$-th cohomology group of $\Sigma_{1} \times \Sigma_{2}$ with real coefficient. The following is also well known. Let $M$ be in general a complex manifold 
and let $W$ be an analytic subvariety of pure dimension $k$, and let $R(W)$ be given as before. Let $D_{c}^{j}(M)$ denote the set of all real valued differential $j$-forms on $M$ with compact support. Then for all $\omega \in D_{c}^{2 k}(M), \int_{R(W)} \omega$ is well defined and for any $\omega \in D_{c}^{2 k-1}(M), \int_{R(W)} d \omega=0$, where the integrations are always taken with respect to the natural orientations on $M-R(W)$ induced from the orientation on $M$. For the details, see [10] for the first half; as for the 2nd half, this will be shown by the extended version of Stokes' theorem [25].

Let $\eta_{1}$ and $\eta_{2}$ be the normal contact structures on $\Sigma_{1}$ and $\Sigma_{2}$ as in Theorem 2 in Part I, respectively. As before, we denote by $\Theta_{1}$ and $\Theta_{2}$ the kernel subbundles of $\eta_{1}$ and $\eta_{2}$, respectively. Let $\xi_{1}$ and $\xi_{2}$ be the velocity vector fields of the $S^{1}$-orbits on $\Sigma_{1}$ and $\Sigma_{2}$, respectively. Then we know that $\xi_{1}$ and $\Theta_{1}$ (or $\xi_{2}$ and $\Theta_{2}$ ) span $T \Sigma_{1}$ (or $T \Sigma_{2}$ ). Now define a 1-form $\omega$ on $\Sigma_{1} \times \Sigma_{2}$ as follows.

$$
\begin{array}{cc}
\omega\left(\xi_{i}\right)=\eta_{i}\left(\xi_{i}\right)=1 \quad(i=1,2) \\
\omega\left(\Theta_{i}\right)=\eta_{i}\left(\Theta_{i}\right)=0 \quad(i=1,2) .
\end{array}
$$

If we define 1 -forms $\bar{\eta}_{1}$ and $\bar{\eta}_{2}$ on $\Sigma_{1} \times \Sigma_{2}$ by

$$
\begin{aligned}
& \bar{\eta}_{i}\left(T \Sigma_{i}\right)=\eta_{i}\left(T \Sigma_{i}\right) \quad(i=1,2) \\
& \bar{\eta}_{i}\left(T \Sigma_{j}\right)=0 \quad(i, j=1,2 \text { and } \quad i \neq j),
\end{aligned}
$$

then

$$
\omega=\bar{\eta}_{1}+\bar{\eta}_{2}
$$

Clearly $\omega$ is a smooth 1 -form. Thus,

$$
d \omega=d \bar{\eta}_{1}+d \bar{\eta}_{2}
$$

and

$$
\begin{aligned}
(d \omega)^{l} & =\underbrace{d \omega \wedge \cdots \wedge d \omega}_{l \text {-times }}=\left(d \bar{\eta}_{1}+d \bar{\eta}_{2}\right)^{l} \\
& =\frac{\left(d \bar{\eta}_{1}+d \bar{\eta}_{2}\right) \wedge \cdots \wedge\left(d \bar{\eta}_{1}+d \bar{\eta}_{2}\right)}{l \text {-times }} \\
& =\sum_{i=1}^{l}\left(\begin{array}{l}
l \\
i
\end{array}\right)\left(d \bar{\eta}_{1}\right)^{i} \wedge\left(d \bar{\eta}_{2}\right)^{l-i} .
\end{aligned}
$$

Note here that $d \bar{\eta}_{1}$ and $d \bar{\eta}_{2}$ are 2-forms, so they commute with the wedge product.

LEMMA 2. $\int_{R(W)}(d \omega)^{k}=0$. 
Proof. As we explained, this integral is well defined. Since $\Sigma_{1} \times \Sigma_{2}$ is compact, any differential form on $\Sigma_{1} \times \Sigma_{2}$ can be considered as a form with compact support. Now define a $(2 k-1)$-form $\phi$ on $\Sigma_{1} \times \Sigma_{2}$ by $\phi=$ $\omega \wedge(d \omega)^{k-1}$. Then $d \phi=d \omega \wedge(d \omega)^{k-1}=(d \omega)^{k}$. By the above generalized Stokes' theorem, $\int_{R(W)}(d \omega)^{k}=\int_{R(W)} d \dot{\phi}=0$.

q.e.d.

Let $P_{i}: T\left(\Sigma_{1} \times \Sigma_{2}\right) \rightarrow \Theta_{i}(i=1,2)$ be the natural projection defined by

$$
\begin{aligned}
P_{i}\left(T \Sigma_{j}\right) & =0 \quad(i \neq j) \\
P_{i}\left(\xi_{i}\right) & =0 \\
P_{i}(X) & =X, X \in \Theta_{i} \quad(i, j=1,2) .
\end{aligned}
$$

Now define a natural projection $P: T\left(\Sigma_{1} \times \Sigma_{2}\right)=T \Sigma_{1} \hat{\oplus} T \Sigma_{2} \rightarrow \Theta_{1} \hat{\oplus} \Theta_{2}$ by the exterior direct sum of $P_{1}$ and $P_{2}$, i.e., $P=P_{1} \hat{\oplus} P_{2}$; therefore for any $X=X_{1} \hat{\oplus} X_{2}$ in $T\left(\Sigma_{1} \times \Sigma_{2}\right), P(X)=P_{1}\left(X_{1}\right) \hat{\oplus} P_{2}\left(X_{2}\right)$. We also denote by $T\left(\Sigma_{1} \times \Sigma_{2}\right)_{(x, y)}$ the tangent space of $\Sigma_{1} \times \Sigma_{2}$ at $(x, y)$.

Lemma 3. Let $\bar{\eta}_{i}(i=1,2)$ be given as before. Then we have,

i) $d \bar{\eta}_{i}(X, Y)=0$ if $X \in T \Sigma_{i}$ and $Y \in T \Sigma_{j}(j \neq i), i, j=1,2$, or $X, Y \in T \Sigma_{j}(j \neq i)$.

ii) $d \bar{\eta}_{i}\left(\xi_{j}, X\right)=0$ for all $X \in T\left(\Sigma_{1} \times \Sigma_{2}\right), i, j=1,2$.

iii) $d \bar{\eta}_{i}(X, Y)=d \eta_{i}(X, Y)$ if $X$ and $Y \in T \Sigma_{i}(i=1,2)$.

Proof. As $d \bar{\eta}_{i}$ is a tensor, we can assume that $X$ is independent of the points in $\Sigma_{j}$ and $Y$ is independent of $\Sigma_{i}$. Then

$$
d \bar{\eta}_{i}(X, Y)=X \bar{\eta}_{i}(Y)-Y \bar{\eta}_{i}(X)-\bar{\eta}_{i}[X, Y]=0-Y \eta_{i}(X)-0=0 .
$$

This proves i), and ii) can be shown in the same way. As for iii),

$$
\begin{aligned}
d \bar{\eta}_{i}(X, Y) & =X \bar{\eta}_{i}(Y)-Y \bar{\eta}_{i}(X)-\bar{\eta}_{i}[X, Y] \\
& =X \eta_{i}(Y)-Y \eta_{i}(X)-\eta_{i}[X, Y]=d \eta_{i}(X, Y) . \quad \text { q.e.d. }
\end{aligned}
$$

Lemma 4. Let $X_{1}, \cdots, X_{l}, J X_{1}, \cdots, J X_{l}$ be any $2 l$ linearly independent vectors in $T\left(\Sigma_{1} \times \Sigma_{2}\right)_{(x, y)}$, where $J$ is the complex structure of $\Sigma_{1} \times \Sigma_{2}$. Furthermore, assume that they are ordered in the above way. Then we have,

$$
(d \omega)^{l}\left(X_{1}, \cdots, X_{l}, J X_{1}, \cdots, J X_{l}\right)=(-1)^{l l(-1) / 2} \sum_{i=1}^{l} N_{i}\left(\frac{1}{\omega_{1}\left(\xi_{1}\right)}\right)^{i}\left(\frac{1}{\omega_{2}\left(\xi_{2}\right)}\right)^{l-i} .
$$

$N_{i}(0 \leqq i \leqq l)$ is a non-negative constant which will be given in the following proof, and $\omega_{i}$ and $\xi_{i}(i=1,2)$ are given as before.

Proof. Let us denote by $\operatorname{Sp}(X)$ the span of $\left\{X_{1}, \cdots, X_{l}, J X_{1}, \cdots, J X_{l}\right\}$. $\mathrm{Sp}(X)$ is a complex vector subspace of $T\left(\Sigma_{1} \times \Sigma_{2}\right)_{(x, y)}$ of complex dimension 
$l$, where a complex vector space of dimension $l$ means a real $2 l$ dimensional subspace of $T\left(\Sigma_{1} \times \Sigma_{2}\right)_{(x, y)}$ which is invariant under the complex structure $J$. Now for the sake of convention, denote $X_{1}=E_{1}, \cdots, X_{l}=E_{l} ; E_{l+1}=$ $J X_{1}, \cdots, E_{2 l}=J X_{l}$. Then by the above (*), we have,

$$
\begin{aligned}
(d \omega)^{l}\left(X_{1}, \cdots, X_{l} ; J X_{1}, \cdots, J X_{l}\right) & \\
= & \sum_{i=1}^{l}\left(\begin{array}{l}
l \\
i
\end{array}\right)\left(d \bar{\eta}_{1}\right)^{i} \wedge\left(d \bar{\eta}_{2}\right)^{l-i}\left(X_{1}, \cdots, X_{l} ; J X_{1}, \cdots, J X_{l}\right) \\
= & \sum_{i=1}^{l}\left(\begin{array}{l}
l \\
i
\end{array}\right)\left[\sum _ { \sigma \in \mathfrak { S } } ( \operatorname { s g n } \sigma ) \left(d \bar{\eta}_{1}\left(E_{\sigma(1)}, E_{\sigma(2)}\right) \cdots d \bar{\eta}_{1}\left(E_{\sigma(2 i-1)}, E_{\sigma(2 i)}\right)\right.\right. \\
& \times\left(d \bar{\eta}_{2}\left(E_{\sigma(2 i+1)}, E_{\sigma(2 i+2)}\right) \cdots d \bar{\eta}_{2}\left(E_{\sigma(2 l-1)}, E_{\sigma(2 l)}\right)\right] .
\end{aligned}
$$

Here $\subseteq$ is the set of all the permutations of $2 l$ letters $(1, \cdots, 2 l)$, and $\operatorname{sgn} \sigma$ is the $\operatorname{sign}$ of $\sigma$. Denote by $\operatorname{Sp}\left(\xi_{1}, \xi_{2}\right)$ the span of $\xi_{1}$ and $\xi_{2}$, and assume that $\operatorname{Sp}(X)$ is not transversal to $\operatorname{Sp}\left(\xi_{1}, \xi_{2}\right)$. In other words, $\operatorname{Sp}\left(\xi_{1}, \xi_{2}\right) \subset \operatorname{Sp}(X)$. Note here that $\operatorname{Sp}\left(\xi_{1}, \xi_{2}\right)$ is a complex line in $T\left(\Sigma_{1} \times \Sigma_{2}\right)_{(x, y)}$. Then we can take a new ordered basis $\left\{Y_{1}, \cdots, Y_{l} ; J Y_{1}, \cdots, J Y_{l}\right\}$ for $\operatorname{Sp}(X)$ such that $Y_{1}=\xi_{1}$ and $J Y_{1}=\xi_{2}$. Since $\left\{Y_{i}, J Y_{i}\right\}_{i=1}^{l}$ forms a basis, we have for $1 \leqq i \leqq l$,

$$
\begin{aligned}
X_{i} & =a_{1 i} Y_{1}+\cdots+a_{l i} Y_{l}+b_{1 i} J Y_{1}+\cdots+b_{l i} J Y_{l} \\
J X_{i} & =-b_{1 i} Y_{1}+\cdots+\left(-b_{l i} Y_{l}\right)+a_{1 i} J Y_{1}+\cdots+a_{l i} J Y_{l} .
\end{aligned}
$$

The coefficient matrix $L$ is written as

$$
L=\left(\begin{array}{rr}
A & -B \\
B & A
\end{array}\right),
$$

where $A=\left(a_{i j}\right)$ and $B=\left(b_{i j}\right), 1 \leqq i, j \leqq l$. As is well known, the determinant of $L$, say $|L|$, is positive. By elementary exterior algebra, we have

$$
(d \omega)^{l}\left(X_{1}, \cdots, X_{l}, J X_{1}, \cdots, J X_{l}\right)=|L|(d \omega)^{l}\left(Y_{1}, \cdots, Y_{l}, J Y_{1}, \cdots, J Y_{l}\right) .
$$

By the similar expression to $(* *)$ for the second half, each summand of that expression must contain a term of the form $\pm d \bar{\eta}_{1}\left(\xi_{1}, *\right)$ or $\pm d \bar{\eta}_{2}\left(*, \xi_{2}\right)$. By Lemma 3, these terms are zero; hence, $(d \omega)^{l}\left(X_{1}, \cdots, X_{l}, J X_{1}, \cdots, J X_{l}\right)=$ 0 . Therefore, we can automatically assume that $\operatorname{Sp}(X)$ is transversal to $\operatorname{Sp}\left(\xi_{1}, \xi_{2}\right)$. In this case $P$ restricted to $\operatorname{Sp}(X)$ is a complex isomorphism of $\operatorname{Sp}(X)$ onto its image $P \operatorname{Sp}(X)$ under $P$. $P \operatorname{Sp}(X)$ is contained in $\Theta_{1} \hat{\oplus} \Theta_{2}$ and has complex dimension $l$. Let us denote by $P_{i}(i=1,2)$ the restriction of $P_{i}$ to $P \operatorname{Sp}(X)$ for the sake of convenience. Denote the 
kernel of $P_{i}$ by $\operatorname{Ker} P_{i}(i=1,2)$. Clearly, $\operatorname{Ker} P_{i}(i=1,2)$ is orthogonal to each other with respect to the Riemannian metric $g$, where $g$ is given as the product metric of $g_{i}(i=1,2)$ which is the associated Riemannian metric on $\Sigma_{i}(i=1,2)$. Now let $P \operatorname{Sp}(X)$ be as before, and let $W$ be the orthogonal complement of $\left(\operatorname{Ker} P_{2}\right) \oplus\left(\operatorname{Ker} P_{1}\right)$ in $P \operatorname{Sp}(X)$. Then $P \operatorname{Sp}(X)$ can be written as the orthogonal direct sum $\left(\operatorname{Ker} P_{2}\right) \oplus W \oplus\left(\operatorname{Ker} P_{1}\right)$. Notice here that $P_{1}$ restricted to $\left(\operatorname{Ker} P_{2}\right) \oplus W$ is an isomorphism, and similarly $P_{2}$ restricted to $W \oplus \operatorname{Ker} P_{1}$ is an isomorphism. Furthermore, $P_{1}\left(\operatorname{Ker} P_{2}\right)$ and $P_{1}(W)$ are orthogonal to each other in $\Theta_{1}$, and similarly $P_{2}\left(\operatorname{Ker} P_{1}\right)$ and $P_{2}(W)$ are orthogonal to each other in $\Theta_{2}$. Denote by $p, q$, and $r$ the complex dimensions of $\left(\operatorname{Ker} P_{2}\right), W$ and $\left(\operatorname{Ker} P_{1}\right)$, respectively. Let $\left\{Z_{1}, \cdots Z_{p}, J Z_{1}, \cdots, J Z_{p}\right\}$ be an ordered unitary basis for $P_{1}\left(\operatorname{Ker} P_{2}\right)$, and similarly $\left\{Z_{p+1}, \cdots, Z_{p+q}, J Z_{p+1}, \cdots, J Z_{p+q}\right\}$ and $\left\{Z_{p+q+1}, \cdots, Z_{p+q+r=l}, J Z_{p+q+1}\right.$, $\left.\cdots, J Z_{p+q+r=l}\right\}$ be ordered unitary bases for $P_{1}(W)$ and $P_{2}\left(\operatorname{Ker} P_{1}\right)$, respectively. Define $2 l$ vectors in $P \operatorname{Sp}(X)$ denoted by $\left\{Y_{1}, \cdots, Y_{l}, J Y_{1}, \cdots, J Y_{l}\right\}$ in such a way that $P_{1}\left(Y_{1}\right)=Z_{1}, \cdots, P_{1}\left(Y_{p}\right)=Z_{p}, P_{1}\left(Y_{p+1}\right)=Z_{p+1}, \cdots$, $P_{1}\left(Y_{p+q}\right)=Z_{p+q}, P_{2}\left(Y_{p+q+1}\right)=Z_{p+q+1}, \cdots, P_{2}\left(Y_{l}\right)=Z_{l}$ and $P_{1}\left(J Y_{1}\right)=J P_{1}\left(Y_{1}\right)=$ $J Z_{1}, \cdots, P_{1}\left(J Y_{p}\right)=J Z_{p}, P_{1}\left(J Y_{p+1}\right)=J Z_{p+1}, \cdots, P_{1}\left(J Y_{p+q}\right)=J Z_{p+q}, P_{2}\left(J Y_{p+q+1}\right)=$ $J Z_{p+q+1}, \cdots, P_{2}\left(J Y_{l}\right)=J Z_{l}$. Since $P_{1} \mid \operatorname{Ker} P_{2} \oplus W$ is a complex isomorphism, $\left\{Y_{1}, \cdots, Y_{l}, J Y_{1}, \cdots, J Y_{l}\right\}$ forms an ordered complex basis for $P \operatorname{Sp}(X)$, and the orientation induced by the order coincides with the induced orientation from the natural orientation of the ambient space. Now let $\left\{\bar{X}_{1}, \cdots, \bar{X}_{l}, J \bar{X}_{1}, \cdots, J \bar{X}_{l}\right\}$ be given as follows. $P\left(\bar{X}_{1}\right)=Y_{1}, \cdots, P\left(\bar{X}_{l}\right)=$ $Y_{l}, P\left(J \bar{X}_{1}\right)=J Y_{1}, \cdots, P\left(J \bar{X}_{l}\right)=J Y_{l}$. Since $P: \mathrm{Sp}(X) \rightarrow P \mathrm{Sp}(X)$ is a complex isomorphism, $\left\{\bar{X}_{1}, \cdots, \bar{X}_{l}, J \bar{X}_{1}, \cdots, J \bar{X}_{l}\right\}$ in the given order forms an ordered basis for $\operatorname{Sp}(X)$ with the natural induced orientation. Denote it in the same order as follows: $E_{1}=\bar{X}_{1}, \cdots, E_{l}=\bar{X}_{l}, E_{l+1}=J \bar{X}_{1}, \cdots$, $E_{2 l}=J \bar{X}_{l}$. In order to compute $(d \omega)^{l}$ at $\left(E_{1}, \cdots, E_{l}, E_{l+1}, \cdots, E_{2 l}\right)$, we make use of the expression $(* *)$.

To be more precise, put $E_{i}=\left(a_{i} \xi_{1} \oplus P_{1} P\left(E_{i}\right)\right) \hat{\oplus}\left(b_{i} \xi_{2} \oplus P_{2} P\left(E_{i}\right)\right), i=$ $1, \cdots, 2 l$. By Lemma 3 , we have for $1 \leqq i, j \leqq 2 l$,

$$
\begin{aligned}
(* * *) \quad d \bar{\eta}_{1}\left(E_{i}, E_{j}\right)= & d \bar{\eta}_{1}\left(\left(a_{i} \xi_{1} \oplus P P_{1} P\left(E_{i}\right)\right) \hat{\oplus}\left(b_{i} \xi_{2} \oplus P_{2} P\left(E_{i}\right)\right),\right. \\
& \left.\left(a_{j} \xi_{1} \oplus P_{1} P\left(E_{j}\right)\right) \hat{\oplus}\left(b_{j} \xi_{2} \oplus P_{2} P\left(E_{j}\right)\right)\right) \\
= & d \bar{\eta}_{1}\left(a_{i} \xi_{1} \oplus P_{1} P E_{i}, a_{j} \xi_{1} \oplus P_{1} P E_{j}\right)+d \bar{\eta}_{1}\left(a_{i} \xi_{1} \oplus P_{1} P E_{i},\right. \\
& \left.b_{j} \xi_{2} \oplus P_{2} P E_{j}\right) \\
& +d \bar{\eta}_{1}\left(b_{i} \xi_{2} \oplus P_{2} P E_{i}, a_{j} \xi_{1} \oplus P_{1} P E_{j}\right)+d \bar{\eta}_{1}\left(b_{i} \xi_{2} \oplus P_{2} P E_{i},\right. \\
& \left.\quad b_{j} \xi_{2} \oplus P_{2} P E_{j}\right) \\
= & d \bar{\eta}_{1}\left(a_{i} \xi_{1} \oplus P_{1} P E_{i}, a_{j} \xi_{1} \oplus P_{1} P E_{j}\right) \\
= & d \eta_{1}\left(P_{1} P E_{i}, P_{1} P E_{j}\right) .
\end{aligned}
$$


Similarly,

$$
d \bar{\eta}_{2}\left(E_{i}, E_{j}\right)=d \eta_{2}\left(P_{2} P E_{i}, P_{2} P E_{j}\right), \quad 1 \leqq i, j \leqq 2 l .
$$

Therefore, for $1 \leqq i \leqq 2 l$, each summand of the expression $(* *)$ becomes

$$
\begin{aligned}
(\operatorname{sgn} \sigma) d \bar{\eta}_{1}\left(E_{\sigma(1)}, E_{o(2)}\right) & \cdots d \bar{\eta}_{1}\left(E_{o(2 i-1)}, E_{o(2 i)}\right) d \bar{\eta}_{2}\left(E_{o(2 i+1)}, E_{o(2 i+2)}\right) \\
& \cdots d \bar{\eta}_{2}\left(E_{o(22-1)}, E_{o(2 l)}\right) \\
= & (\operatorname{sgn} \sigma)\left(d \eta_{1}\left(P_{1} P E_{\sigma(1)}, P_{1} P E_{o(2)}\right) \cdots d \eta_{1}\left(P_{1} P E_{o(2 i-1)}, P_{1} P E_{\sigma(2 i)}\right)\right) \\
& \times\left(d \eta_{2}\left(P_{2} P E_{\sigma(2 i+1)}, P_{2} P E_{o(2 i+2)}\right) \cdots d \eta_{2}\left(P_{2} P E_{o(2 l-1)}, P_{2} P E_{\sigma(2))}\right) .\right.
\end{aligned}
$$

Since $P_{1} P E_{i}=0$ if $P E_{i}$ belongs to $\operatorname{Ker} P_{1}$, the term (\#) vanishes unless all $E_{\sigma(1)}, \cdots, E_{\sigma(2 i)}$ satisfy that $P E_{\sigma(1)}, \cdots, P E_{\sigma(2 i)}$ belong to $\operatorname{Ker} P_{2} \oplus W$. Hence, we treat the case mentioned above. In other words, $P_{1} P E_{o(1)}, \cdots$, $P_{1} P E_{o(2 i)}$ belong to $\operatorname{Ker} P_{2} \oplus W$. By the definition, $\left\{Z_{1}, \cdots, Z_{p+q}, J Z_{1}, \cdots\right.$, $\left.J Z_{p+q}\right\}$ forms an ordered unitary basis for $\operatorname{Ker} P_{2} \oplus W$. Thus for a fixed $\sigma \in \mathfrak{S}$, the span of $P_{1} P E_{\sigma(1)}$ through $P_{1} P E_{o(22)}$ is a subspace of $\operatorname{Ker} P_{2} \oplus W$. Now recall b) in Lemma 5 , then we have $d \eta_{1}(X, Y)=\langle J X, Y\rangle / \omega_{1}\left(\xi_{1}\right)$ where \langle\rangle is the induced metric on $\Theta_{1}$ from the standard metric on $C^{n+1}$. This metric also coincides with the associated Riemannian metric on $\theta_{1}$, since we consider $\Sigma_{1}$ as the intersection of the variety and the standard hypersphere. As remarked before, $P_{1} P E_{\sigma(1)}, \cdots, P_{1} P E_{\sigma(2 i)}$ are among the unitary basis $\left\{Z_{1}, \cdots, Z_{p+q}, J Z_{1}, \cdots, J Z_{p+q}\right\}$ for $\operatorname{Ker} P_{2} \oplus W$. This means that $d \eta_{1}\left(P_{1} P E_{\sigma(2 j-1)}, P_{1} P E_{o(2 j)}\right)=0$ unless $P_{1} P E_{\sigma(2 j-1)}= \pm J P_{1} P E_{\sigma(2 j)}(j=1, \cdots, l)$. In other words, $(\sharp) \neq 0$ only when $\sigma(1)=\sigma(2) \pm l, \cdots, \sigma(2 i-1)=\sigma(2 i) \pm l$. Now let $\sigma$ be the permutation for which (\#) does not vanish. Denote $\sigma$ by $\sigma=\left(\begin{array}{l}1,2, \cdots, 2 i, 2 i+1, \cdots, 2 l \\ \sigma(1), \cdots, \sigma(2 i), \sigma(2 i+1), \cdots, \sigma(2 l)\end{array}\right)$. Let $\mu$ be the permutation of $2 i$ ordered letters $\{\sigma(1), \cdots, \sigma(2 i)\}$ such that $\mu$ permutes $\{\sigma(1), \cdots, \sigma(2 i)\}$ into the ordered set $\{\mu(1), \cdots, \mu(2 i)\}$ with $\mu(1)<\mu(2)<\cdots<\mu(2 i)$. Similarly, let $\tau$ be the permutation of $2(l-i)$ letters $\{\sigma(2 i+1), \cdots, \sigma(2 l)\}$ such that $\tau$ permutes $\{\sigma(2 i+1), \cdots, \sigma(2 l)\}$ into $\{\tau(1), \cdots, \tau(2(l-i))\}$ with $\tau(1)<\tau(2)<\cdots<\tau(2(l-i))$. Let $\alpha$ be the number of pairs $(\sigma(2 j-1)$, $\sigma(2 j))(j=1, \cdots, i)$ such that $\sigma(2 j-1)=\sigma(2 j)+l$, and let $\beta$ be a number of transpositions to get $\tau$. Then after a lengthy but elementary observation of permutations involving reshuffling the indices, we obtain that

$$
\operatorname{sgn} \sigma=(-1)^{\alpha+\beta+i(2 l-i-1) / 2}=(-1)^{\alpha+i(2 l-i-1) / 2} \operatorname{sgn} \tau .
$$

Next consider the set $\mathfrak{S}_{\sigma}$ of permutations in $\subseteq$ such that each member of $\mathfrak{S}_{\sigma}$ coincides with $\sigma$ on the first $2 i$ letters. Then we have,

$$
\begin{gathered}
\sum_{\rho \in S_{\sigma}}(\operatorname{sgn} \rho) d \bar{\eta}_{1}\left(E_{\rho(1)}, E_{\rho(2)}\right) \\
\cdots d \bar{\eta}_{1}\left(E_{\rho(2 i-1)}, E_{\rho(2 i)}\right) d \bar{\eta}_{2}\left(E_{\rho(2 i+1)}, E_{\rho(2 i+2)}\right) \\
\cdots d \bar{\eta}_{2}\left(E_{\rho(2 l-1)}, E_{\rho(2 l)}\right)
\end{gathered}
$$




$$
\begin{aligned}
= & \sum_{\rho \in \mathbb{E}_{\sigma}}(-1)^{\alpha+i(2 l-i-1) / 2}(\operatorname{sgn} \tau)\left[\left(d \bar{\eta}_{1}\left(E_{\rho(1)}, E_{\rho(2)}\right) \cdots d \bar{\eta}_{1}\left(E_{\rho(2 i-1)}, E_{\rho(2 i)}\right)\right)\right. \\
& \left.\times\left(d \bar{\eta}_{2}\left(E_{\rho(2 i+1)}, E_{\rho(2 i+2)}\right) \cdots d \bar{\eta}_{2}\left(E_{\rho(2 l-1)}, E_{\rho(2 l)}\right)\right)\right] .
\end{aligned}
$$

Here $\tau$ is the permutation of the last $2(l-i)$ letters $\sigma(2 i+1), \cdots, \sigma(2 l)$. Taking into account that for any $\rho \in \mathbb{S}_{\sigma}$ the first $2 i$-letters are always given as $\sigma(1), \cdots, \sigma(2 i)$, we can identify any such element $\rho \in \mathfrak{S}_{o}$ with the permutation of the last $2(l-i)$ letters $(\sigma(2 i+1), \cdots, \sigma(2 l))$. There are exactly the same number of permutations in $\mathfrak{S}_{\sigma}$ as the set of all permutations of $2(l-i)$ letters. Denote the latter by $\mathfrak{S}^{\prime}$. Here any element of $\mathscr{S}^{\prime}$ is identified with $\tau^{-1}$, where $\tau$ is given such as before. In other words, $\tau$ transforms the last $2(l-i)$ letters of any permutation $\rho$ in $\mathscr{S}_{\sigma}$ into $(\tau(1), \cdots, \tau(2(l-i))$ such that $\tau(1)<\tau(2)<\cdots<\tau(2(l-i))$. Also note here that $\operatorname{sgn} \tau=\operatorname{sgn} \tau^{-1}$. With these in mind we have,

$$
\begin{aligned}
(\# \#)= & \sum_{\tau \in E_{\sigma}}(-1)^{\alpha+i(2 l-i-1) / 2}(\operatorname{sgn} \tau)\left[d \bar{\eta}_{1}\left(E_{\sigma(1)}, E_{\sigma(2)}\right) \cdots d \bar{\eta}_{1}\left(E_{\sigma(2 i-1)}, E_{\sigma(2 i)}\right)\right. \\
& \left.\times d \bar{\eta}_{2}\left(E_{\tau^{-1}(1)}, E_{\tau^{-1}(2)}\right) \cdots d \bar{\eta}_{2}\left(E_{\tau^{-1}(2(l-i)-1)}, E_{\tau^{-1}(2(l-i))}\right)\right] \\
= & (-1)^{\alpha+i(2 l-i-1) / 2}\left[d \eta_{1}\left(P_{1} P E_{\sigma(1)}, P_{1} P E_{o(2)}\right) \cdots d \eta_{1}\left(P_{1} P E_{\sigma(2 i-1)}, P_{1} P E_{\sigma(2 i)}\right)\right] \\
& \times\left[\sum _ { \tau \in \mathbb { S } ^ { \prime } } ( \operatorname { s g n } \tau ^ { - 1 } ) d \eta _ { 2 } ( P _ { 2 } P E _ { \tau ^ { - 1 ( 1 ) } } , P _ { 2 } P E _ { \tau ^ { - 1 ( 2 ) } } ) \cdots d \eta _ { 2 } \left(P_{2} P E_{\tau^{-1}(2(l-i)-1)},\right.\right. \\
& \left.P_{2} P E_{\left.\tau^{-1(2(l-i))}\right)}\right) .
\end{aligned}
$$

By the definition of $\alpha$, applying the same argument as in the proof of Theorem 2 in Part I,

$$
(-1)^{\alpha} d \eta_{1}\left(P_{1} P E_{\sigma(1)}, P_{1} P E_{\sigma(2)}\right) \cdots d \eta_{1}\left(P_{1} P E_{o(2 i-1)}, P_{1} P E_{\sigma(2 i)}\right)=\left(1 / \omega_{1}\left(\xi_{1}\right)\right)^{i} .
$$

Also the term inside the second bracket $[\cdots]$ in the above (\#\#) is equal to $\left(d \eta_{2}\right)^{l-i}\left(P_{2} P E_{\tau(1)}, \cdots, P_{2} P E_{\tau(2(l-i))}\right)$. Recall that $\left(P_{2} P E_{\tau(1)}, \cdots, P_{2} P E_{\tau(2(l-i))}\right)$ forms an ordered complex basis for their span in $P_{2}\left(W \oplus \operatorname{Ker} P_{1}\right)$, since $P_{2} \mid W \oplus \operatorname{Ker} P_{1}$ is a complex isomorphism and $\left\{P E_{\tau(1)}, \cdots, P E_{\tau(2\{l-i)\}}\right\}$ forms a complex basis for their span which has the natural induced orientation. Note here that $J P_{2} P E_{\tau(1)}=P_{2} P E_{\tau(2(l-i)+1)}, \cdots, J P_{2} P E_{\tau(l-i)}=P_{2} P E_{\tau(2(l-i))}$. Now let $\left(e_{1}, \cdots, e_{l-i}, J e_{1}, \cdots, J e_{l-i}\right)$ be an ordered unitary basis for the span of $\left(P_{2} P E_{\tau(1)}, \cdots, P_{2} P_{\tau(2(l-i))}\right)$ with the induced orientation. Then there is a non-singular $2(l-i) \times 2(l-i)$ matrix of the form $\left(\begin{array}{ll}A & -B \\ B\end{array}\right)_{\sigma}$ relating $\left(P_{2} P E_{\tau(1)}, \cdots, P_{2} P E_{\tau(2(l-i))}\right)$ to $\left(e_{1}, \cdots, e_{l-i}, J e_{1}, \cdots, J e_{l-i}\right)$. By elementary facts on exterior algebra, we have

$$
(\# \#)=(-1)^{i(2 l-i-1) / 2}\left(1 / \omega_{1}\left(\xi_{1}\right)\right)^{i}\left|\begin{array}{rr}
A & -B \\
B & A
\end{array}\right|_{o}\left(d \eta_{2}\right)^{l-i}\left(e_{1}, \cdots, e_{l-i}, J e_{1}, \cdots, J e_{l-i}\right) .
$$

As in the beginning of this proof, we can easily compute that

$$
\left(d \eta_{2}\right)^{l-i}\left(e_{1}, \cdots, e_{l-i}, J e_{1}, \cdots, J e_{l-i}\right)=(-1)^{(l-i)(l-i-1) / 2} K_{\sigma}\left(1 / \omega_{2}\left(\xi_{2}\right)\right)^{l-i},
$$


where $K_{\sigma}$ is the number of non-vanishing terms which appear as the summands of $\left(d \eta_{2}\right)^{l-i}\left(e_{1}, \cdots, e_{l-i}, J e_{1}, \cdots, J e_{l-i}\right)$. Thus we have

$$
\begin{aligned}
& \text { (\#\#) }=(-1)^{\{i(2 l-i-1)+(l-i)(l-i-1)\} / 2} K_{\sigma}\left|\begin{array}{rr}
A & -B \\
B & A
\end{array}\right|_{\sigma}\left(1 / \omega_{1}\left(\xi_{1}\right)\right)^{i}\left(1 / \omega_{2}\left(\xi_{2}\right)\right)^{l-i} \\
& =(-1)^{l(l-1) / 2} K_{\sigma}\left|\begin{array}{rr}
A & -B \\
B & A
\end{array}\right|_{\sigma}\left(1 / \omega_{1}\left(\xi_{1}\right)\right)^{i}\left(1 / \omega_{2}\left(\xi_{2}\right)\right)^{l-i} .
\end{aligned}
$$

Next let $\sigma$ denote a member in $\subseteq$ such that $d \bar{\eta}_{1}\left(E_{\sigma(1)}, E_{\sigma(2)}\right) \cdots d \bar{\eta}_{1}\left(E_{\sigma(2 i-1)}\right.$, $\left.E_{\sigma(2 i)}\right) \neq 0$. Then we have

$$
\begin{aligned}
\sum_{\sigma \in \mathcal{S}}(\text { sng } \sigma) & \left(d \bar{\eta}_{1}\left(E_{\sigma(1)}, E_{\sigma(2)}\right) \cdots d \bar{\eta}_{1}\left(E_{\sigma(2 i-1)}, E_{\sigma(2 i)}\right)\right) \\
& \times\left(d \bar{\eta}_{2}\left(E_{\sigma(2 i+1)}, E_{\sigma(2 i+2)}\right) \cdots d \bar{\eta}_{2}\left(E_{\sigma(2 l-1)}, E_{\sigma(2 l)}\right)\right) \\
= & \left.(-1)^{l(l-1) / 2} \widetilde{\Sigma}_{i} K_{\sigma}\right|^{A} \quad-\left.\left.B\right|^{A} \quad A\right|_{\sigma}\left(1 / \omega_{1}\left(\xi_{1}\right)\right)^{i}\left(1 / \omega_{2}\left(\xi_{2}\right)\right)^{l-i} .
\end{aligned}
$$

Here the symbol $\widetilde{\Sigma}_{i}$ means to sum over all the permutations in $\mathfrak{S}$ given as follows. Partition $\subseteq$ in such a way that $\sigma$ and $\tau$ belong to the same class if and only if $\sigma(j)=\tau(j)(j=1, \cdots, 2 i)$. Pick a representative from each class and denote the set of all the representatives by $\Sigma_{i}$. Then the summation $\widetilde{\Sigma}_{i}$ must be taken over all the permutations in $\Sigma_{i}$. Note here that $\widetilde{\Sigma}_{i}$ does not depend on the choice of representative. Notice also that in the last expression for (\#\#), only $K_{\sigma}(>0)$ and $\left|\begin{array}{lr}A & -B \\ B & A\end{array}\right|_{\sigma}(>0)$ depend on the choice of $\sigma$ given as above. Therefore, the last expression can be either positive or negative according to the value of $l(l-1) / 2$, dependending only on $l$ but not on $i(1 \leqq i \leqq l)$. Now by the similar expression to $(* *)$ for $\bar{X}_{1}, \cdots, \bar{X}_{l}, J \bar{X}_{1}, \cdots, J \bar{X}_{l}$, we have

$$
\begin{aligned}
(d \omega)^{l}( & \left.\bar{X}_{1}, \cdots, \bar{X}_{l}, J \bar{X}_{1}, \cdots, J \bar{X}_{l}\right) \\
= & \sum_{i=1}^{l}\left(\begin{array}{c}
l \\
i
\end{array}\right)\left[\sum_{\sigma \in \mathscr{S}}(\operatorname{sgn} \sigma)\left(d \bar{\eta}_{1}\left(E_{\sigma(1)}, E_{\sigma(2)}\right) \cdots d \bar{\eta}_{1}\left(E_{\sigma(2 i-1)}, E_{\sigma(2 i)}\right)\right)\right. \\
& \left.\times\left(d \bar{\eta}_{2}\left(E_{\sigma(2 i+1)}, E_{o(2 i+2)}\right) \cdots d \bar{\eta}_{2}\left(E_{\sigma(2 l-1)}, E_{\sigma(2 l)}\right)\right)\right] \\
= & \sum_{i=1}^{l}\left(\begin{array}{c}
l \\
i
\end{array}\right)\left[(-1)^{l(l-1) / 2} \widetilde{\Sigma}_{i} K_{\sigma}\left|\begin{array}{rr}
A & -B \\
B & A
\end{array}\right|_{\sigma}\left(1 / \omega_{1}\left(\xi_{1}\right)\right)^{i}\left(1 / \omega_{2}\left(\xi_{2}\right)\right)^{l-i}\right] \\
= & (-1)^{l(l-1) / 2} \sum_{i=1}^{l}\left[\left(\begin{array}{l}
l \\
i
\end{array}\right) \widetilde{\Sigma}_{i} K_{\sigma}\left|\begin{array}{rr}
A & -B \\
B & A
\end{array}\right|_{\sigma}\right]\left(1 / \omega_{1}\left(\xi_{1}\right)\right)^{i}\left(1 / \omega_{2}\left(\xi_{2}\right)\right)^{l-i} .
\end{aligned}
$$

Here $\widetilde{\Sigma}_{i}$ means the summation given as above. Now since the given vectors $\left\{X_{1}, \cdots, X_{l}, J X_{1}, \cdots, J X_{l}\right\}$ and $\left\{\bar{X}_{1}, \cdots, \bar{X}_{l}, J \bar{X}_{1}, \cdots, J \bar{X}_{l}\right\}$ both form ordered bases for $\operatorname{Sp}(X)$ with the natural induced orientation with respect 
to the complex structure $J$, there is as before a non-singular $2 l \times 2 l$ matrix of the form $\left(\begin{array}{rr}A & -B \\ B & A\end{array}\right)$ with $\left|\begin{array}{rr}A & -B \\ B & A\end{array}\right|>0$ which connect between them. Thus we have

$$
\begin{aligned}
(d \omega)^{l} & \left(X_{1}, \cdots, X_{l}, J X_{1}, \cdots, J X_{l}\right) \\
& =\left|\begin{array}{rr}
A & -B \\
B & A
\end{array}\right|(d \omega)^{l}\left(\bar{X}_{1}, \cdots, \bar{X}_{l}, J \bar{X}_{1}, \cdots, J \bar{X}_{l}\right) \\
& =(-1)^{l(l-1) / 2}\left|\begin{array}{rr}
A & -B \\
B & A
\end{array}\right|_{i=1}^{l}\left[\left(\begin{array}{l}
l \\
i
\end{array}\right) \widetilde{\Sigma}_{i} K_{o}\left|\begin{array}{rr}
A & -B \\
B & A
\end{array}\right|_{\sigma}\right]\left(1 / \omega_{1}\left(\xi_{1}\right)\right)^{i}\left(1 / \omega_{2}\left(\xi_{2}\right)\right)^{l-i}
\end{aligned}
$$

Now let $N_{i}$ be $\left|\begin{array}{rr}A & -B \\ B & A\end{array}\right|\left(\begin{array}{c}l \\ i\end{array}\right)\left\{\widetilde{\Sigma}_{i} K_{o}\left|\begin{array}{rr}A & -B \\ B & A\end{array}\right|_{o}\right\}$. Then we have the desired result.

Going back to the proof of Theorem 4 consider $R(W)$ as a complex submanifold of $\Sigma_{1} \times \Sigma_{2}$ of complex dimension $k$, and consider it is imbedded by $h$ into $\Sigma_{1} \times \Sigma_{2}$. Then

$$
\int_{h(R(W))}(d \omega)^{k}=\int_{R(W)} h^{*}(d \omega)^{k}=\int_{R(W)}\left(d\left(h^{*} \omega\right)\right)^{k},
$$

where $h^{*}$ is the pullback homomorphism induced from $h$. Let us assume that $R(W)$ has the induced orientation from that of $\Sigma_{1} \times \Sigma_{2}$. Now further assume that there is a point $(x, y)$ in $\Sigma_{1} \times \Sigma_{2}$ at which the tangent space $T R(W)_{(x, y)}$ is transversal to the span of $\xi_{1}$ and $\xi_{2}$. In other words, $h_{*}\left(T R(W)_{(x, y)}\right)$ does not contain the span of $\xi_{1}$ and $\xi_{2}$ at $h(x, y)$. According to the definition of integral on manifolds, let us choose a locally finite open covering of $R(W)$ in the following way. Let $\left(U_{0} ; x_{01}, \cdots, x_{0 k}, y_{01}, \cdots, y_{0 k}\right)$ be a local coordinate system about $(x, y)$ such that the coordinate functions satisfy the Cauchy-Riemann equations, i.e., $\partial / \partial y_{0 i}=J\left(\partial / \partial x_{0 i}\right)$ and $\partial / \partial x_{0 j}=-J\left(\partial / \partial y_{0 j}\right)$, where $J$ is the induced complex structure $(0 \leqq i \leqq k$, $0 \leqq j \leqq k)$. Clearly, this coordinate system has the induced orientation. Next let $\left\{\left(U_{i},\left(x_{i 1}, \cdots, x_{i k}, y_{i 1}, \cdots, y_{i k}\right)\right\}_{i=1}^{\infty}\right.$ be a locally finite collection of open sets of $R(W)$ such that $\bigcup_{i=1}^{\infty} U_{i}$ covers $R(W)-U_{0}$ and such that these coordinate functions satisfy the Cauchy-Riemann equations with respect to the induced complex structure $J$. Note here that these neighborhoods have the induced orientation in the natural way. Now let $\left\{\phi_{i}\right\}_{j=0}^{\infty}$ be the partition of unity subordinate to the covering $\left\{U_{j}\right\}_{j=0}^{\infty}$. Then

$$
\begin{aligned}
\int_{R(W)} & \left(h^{*}(d \omega)\right)^{k} \\
= & \sum_{j=0}^{\infty} \int_{U_{j}} \phi_{j} h^{*}(d \omega)^{k}\left(\partial / \partial x_{j 1}, \cdots, \partial / \partial x_{j k}, \partial / \partial y_{j 1}, \cdots, \partial / \partial y_{j k}\right) \\
\quad & \times d x_{j 1} \cdots d x_{j k} d y_{j 1} \cdots d y_{j k},
\end{aligned}
$$


where the summand of the right hand side is the usual Riemannian integral. Since $\phi_{j} \geqq 0(j=0,1, \cdots)$ on $R(W)$ and since by Lemma 4 , either

$$
h^{*}(d \omega)^{k}\left(\partial / \partial x_{j 1}, \cdots, \partial / \partial x_{j k}, \partial / \partial y_{j 1}, \cdots, \partial / \partial y_{j_{k}}\right) \geqq 0 \text { or } \leqq 0,
$$

$\int_{U_{j}} \phi_{j} h^{*}(d \omega)^{l}\left(\partial / \partial x_{j 1}, \cdots, \partial / \partial x_{j k}, \partial / \partial y_{j 1}, \cdots, \partial / \partial y_{j_{k}}\right) d x_{j 1} \cdots d x_{j_{k}} d y_{j_{1}} \cdots d y_{j_{k}}$ is either $\geqq 0$ or $\leqq 0$ according to $l(l-1) / 2$, for all $j=0,1, \ldots$. In particular, again by Lemma 4 , the above integral for $j=0$ is either strictly positive or strictly negative. This can be seen easily as follows. $h^{*}(d \omega)^{k}\left(\partial / \partial x_{01}, \cdots, \partial / \partial x_{0 k}, \partial / \partial y_{01}, \cdots, \partial / \partial y_{0 k}\right)>0$ (or $\left.<0\right)$ at $(x, y)$ by the assumption. By continuity, the value must be positive (or negative) in a neighborhood of $(x, y)$ which has positive measure with respect to $d x_{01} \wedge \cdots \wedge d x_{0 k} \wedge d y_{01} \wedge \cdots \wedge d y_{0 k}$. Thus $\int_{R(W)} h^{*}(d \omega)^{k}>0$ (or $<0$ ), and this is a contradiction to Lemma 2. Therefore, no tangent space of $R(W)$ can be transversal to the span of $\xi_{1}$ and $\xi_{2}$, i.e., it always contains the span of $\xi_{1}$ and $\xi_{2}$. As for the points which belong to the singular locus of $W$, let $W=R(W) \cup W^{k-1} \cup \cdots W^{1} \cup W^{0}$ be the splitting of $W$ by dimension [26, p. 98]. Each $W^{j-1}(j=2, \cdots, k)$ can be considered as a complex submanifold of complex dimension $j-1(>0)$, and it is exactly the set of regular points of the subvariety $W^{j-1} \cup \cdots \cup W^{0}$, i.e., $W^{j-1}=$ $R\left(W^{j-1} \cup \cdots \cup W^{0}\right)$. Thus evaluating $(d \omega)^{j-1}$ on $W^{j-1}$ in the same way as above, we can conclude that each tangent space of each point of $W^{j-1}$ $(j=2, \cdots, k)$ must contain the span of $\xi_{1}$ and $\xi_{2}$. As for $W_{0}$, there are only a finite number of points belonging to $W_{0}$ since $W$ is compact.

Next we show that for each point of $W$ there exists an elliptic curve which is a fiber of $\left(\Sigma_{1} \times \Sigma_{2}, \pi, B\right)$ such that it contains the given point and it is completely contained in $W$. First of all, let us point out that in each $W^{j}(j=1, \cdots, k)$ the distribution defined by assigning to each point in $W^{j}$ the span of $\xi_{1}$ and $\xi_{2}$ at that point is integrable. This can be seen by noticing that $\xi_{1}$ and $\xi_{2}$ are tangent to the fibers everywhere; therefore, the span of $\xi_{1}$ and $\xi_{2}$ is tangent to the fiber. Thus the distribution is involutive. The maximal integral submanifolds of the distributions must be open submanifolds of some fibers. Let $W^{*}$ be a 1-dimensional compact subvariety of $\Sigma_{1} \times \Sigma_{2}$. Then clearly $R\left(W^{*}\right)$ must consist of these maximal integral submanifolds. Any singular sets of $W^{*}$ which consist of a finite number of points are limit points of $R\left(W^{*}\right)$, so they belong to the closure of $R\left(W^{*}\right)$, say $\overline{R\left(W^{*}\right)}$. Let $C R\left(W^{*}\right)$ be a connected component of $R\left(W^{*}\right)$, and let $\overline{C R\left(W^{*}\right)}$ be the closure of it in $W^{*}$. Notice that $\overline{C R\left(W^{*}\right)}-C R\left(W^{*}\right)$ consists of a finite number of points in the singular set of $W^{*}$. Let us call $\overline{C R\left(W^{*}\right)}$ an 
irreducible branch of $W^{*}$ according to Gunning-Rossi [11]. $\overline{C R\left(W^{*}\right)}$ is a subvariety of $W^{*}$ of dimension 1 . It is also clear that $C R\left(W^{*}\right)$ is a maximal integral submanifold of the above distribution. Therefore, $C R\left(W^{*}\right)$ is contained in a fiber $F$. Since $F$ is closed, $F \supset \overline{C R\left(W^{*}\right)}$. If $x$ is ap oint in $C R\left(W^{*}\right) \subset \overline{C R\left(W^{*}\right)}$, since $C R\left(W^{*}\right)$ is an open subset of $F, x$ is an interior point of $\overline{C R\left(W^{*}\right)}$ as a subset of $F$. Let $x$ be a point in $\overline{C R\left(W^{*}\right)}-C R\left(W^{*}\right)$. There are only a finite number of points in this set, so each point is isolated in $F$, too. We want to show that there is an open disk in $F$ which has $x$ as a center and which is entirely contained in $\overline{C R\left(W^{*}\right)}$. Note here that since $\Sigma_{1} \times \Sigma_{2}$ has the Rimannian metric $g$ and since $F$ and $\overline{C R\left(W^{*}\right)}$ are subsets of $\Sigma_{1} \times \Sigma_{2}$, we can naturally assume that these are metric spaces. For any $n$ (positive integers), denote by $D_{n}$ the open disk of radius $1 / n$ in $F$ which has $x$ as its center. Suppose that for any $n>0$, there is always a point in $F-\overline{C R\left(W^{*}\right)}$, say $y_{n}$, in $D_{n}$. Since $x$ is isolated in $F$, we can assume that there is no other point of $\overline{C R\left(W^{*}\right)}-C R\left(W^{*}\right)$ in each $D_{n}$ except for $x$. Since $C R\left(W^{*}\right)$ is connected and since $x$ is a limit point of $C R\left(W^{*}\right)$, there is a certain $n_{0}>0$ such that $D_{n_{0}}-\bar{D}_{n_{0}+1}$ contains a point in $C R\left(W^{*}\right)$ and at least a point $y_{n_{0}} \in$ $F-\overline{C R\left(W^{*}\right)}$. In fact $\left(D_{n_{0}}-\bar{D}_{n_{0}+1}\right) \cap C R\left(W^{*}\right)$ is nonempty open subset of $F$. Thus it must have at least a point in $F-\overline{C R\left(W^{*}\right)}$ as a limit point of $C R\left(W^{*}\right)$. Since $\overline{C R\left(W^{*}\right)}$ is closed (compact), we have a contradiction. Hence there is an open disk $D_{n_{1}}$ (for some $n_{1}>0$ ) which is entirely contained in $\overline{C R\left(W^{*}\right)}$. Thus we have shown that $x$ is an interior point of $\overline{C R\left(W^{*}\right)}$, so $\overline{C R\left(W^{*}\right)}$ is an open subset of $F$ as well as closed; therefore, $\overline{C R\left(W^{*}\right)}$ is $F$ itself. In fact, it can be easily shown that $\overline{C R\left(W^{*}\right)}$ coincides with $F$ as an analytic subvariety by removing singularity of the identity map between them. This process can be applied to any irreducible branch of $W^{*}$ to show that $W^{*}$ is a disjoint union of some fibers. Note here that this argument actually tells us that there is no singular point in 1-dimension analytic subvariety of $\Sigma_{1} \times \Sigma_{2}$. Now applying this observation to our $\bar{W}^{1}$, we have that $W^{1}$ is a disjoint union of some fibers of $\Sigma_{1} \times \Sigma_{2}$. Let $C\left(W^{2}\right)$ be a connected component of $W^{2}$, and let $\overline{C\left(W^{2}\right)}$ be its closure in $W$. Since $W^{2} \cup W^{1} \cup W^{0}$ is closed, $\overline{C\left(W^{2}\right)}-C\left(W^{2}\right)$ is contained either in $W^{1}$ or $W^{0}$. Let $x$ be a point in $C\left(W^{2}\right)$ and let $M$ be the maximal integral submanifold of the above distribution passing through $x$. Then the closure $\bar{M}$ of $M$ is contained in $W^{2} \cup W^{1} \cup W^{0}$. Let us denote by $F$ the fiber that contains $\bar{M}$. If $\bar{M}$ contains a point in $W^{1}$, by the previous observation $F$ must be $M$. This is a contradiction, because $M$ contains a point in $W^{2}$ as an interior point. Note here that $W^{j}(j=0, \cdots, k)$ are mutually disjoint. If $\bar{M}$ consists of points in $W^{2}$ 
and points in $W^{\circ}$, there are basically following two cases. The first is that $\bar{M}$ consists of only points in $W^{2}$. Thus $\bar{M}$ is a compact surface without boundary; hence $\bar{M}$ is open and closed in $F$. This implies $\bar{M}=F$ (and $M=F$ ). In the second case, $\bar{M}-M$ contains at most a finite number of points in $W^{0}$. By the same method used previously, we can actually show that $F=\bar{M}=M$ biholomorphically. Continue in like manner, we can show the desired result in our theorem. Our argument automatically gives us that there is no zero dimensional singular variety of $W$ unless $W$ itself is of 0 dimension. It is well known [11] that if $h: X \rightarrow Y$ is a proper holomorphic mapping from an analytic space $X$ into an analytic space $Y$, then the image $h(X)$ of $X$ under $h$ is an analytic subvariety of $Y$.

Since $\pi: \Sigma_{1} \times \Sigma_{2} \rightarrow B_{1} \times B_{2}$ is holomorphic by Lemma 1 , and since $W$ is compact, $\pi(W)$ is a subvariety of $B_{1} \times B_{2}$; and $\pi$ restricted to $W$ is holomorphic. Thus $(W, \pi \mid W, \pi(W))$ is an analytic fibration. q.e.d.

REMARK 2. a) It is not necessary to assume that $\Sigma_{1}$ and $\Sigma_{2}$ are in general position in order to show that $W$ is fibered by elliptic curves. In this case, however, we do not know whether or not $\pi$ is holomorphic. b) Theorem 4 can be shown by making use of the fact that every irreducible compact analytic subvariety can be triangulated in such a way that each descending chain of singular loci can also be triangulated simultaneously and that it forms a descending chain of simplicial subcomplexes. For the details, see Giesecke [8].

The following is a little more precise result than Theorem 4.

THEOREM 5. Let $M$ be a compact complex manifold of complex dimension $k$ and let $h: M \rightarrow \Sigma_{1} \times \Sigma_{2}$ be a proper mapping from $M$ into $\Sigma_{1} \times \Sigma_{2}$. Then $f(M)$ is fibered by elliptic curves, and the fibration is induced from that of $\Sigma_{1} \times \Sigma_{2}$. In particular, if $h$ is an immersion, $M$ is foliated by elliptic curves. $h$ restricted to each leaf is a covering projection. Furthermore, let $B$ denote the quotient space by the foliation. Then $B$ has the natural structure of an analytic space, and there is a holomorphic mapping $\bar{h}$ from $B$ into $B_{1} \times B_{2}$ such that the following diagram commutes.

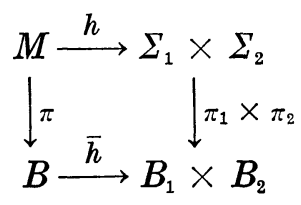

Here $\pi$ is the natural projection, and $\left(\Sigma_{1}, \pi_{1}, B_{1}\right)$ and $\left(\Sigma_{2}, \pi_{2}, B_{2}\right)$ are the fibrations in general position. 
Proof. The proof of Theorem 4 applies verbatim to the first half of the statement because $h(M)$ becomes an analytic subvariety of $\Sigma_{1} \times \Sigma_{2}$. However, we do not have to use the generalized version of Stokes theorem here as we will show. Let $\omega$ and $\phi$ be the same 1 -form and $(2 k-1)$ form as in the proof of the last theorem. Denote by $h^{*}$ the pullback homomorphism and by $h_{*}$ the Jacobian mapping induced from $h$. Consider the integral $\int_{M} h^{*}(d \omega)^{k}$. Using the ordinary Stokes theorem on $M$, we have,

$$
\int_{M} h^{*}(d \omega)^{k}=\int_{M}\left(d h^{*} \omega\right)^{k}=\int_{M} d h^{*} \phi=\int_{\partial M} h^{*} \phi=0 .
$$

Note here that the boundary $\partial M$ of $M$ is empty. This tells us that at each point $x \in M$, there exists a complex vector subspace of $T M_{x}$ which is mapped onto the span of $\xi_{1}$ and $\xi_{2}$ at $h(x)$ by $h_{*}$. Otherwise, we would have a contradiction as in the proof of Theorem 4. However, the above observation gives a little more information than just applying the argument in Theorem 4 directly. Now let $h: M \rightarrow \Sigma_{1} \times \Sigma_{2}$ be a holomorphic immersion. Then by the above observation, for each $x \in M$, there exists a unique complex line in $T M_{x}$ which is mapped isomorphically onto the span of $\xi_{1}$ and $\xi_{2}$ at $h(x)$ under $h_{*}$. Assign to each $x \in M$ that complex line, say $D_{x}$. Then since $h$ is a holomorphic immersion the assignment $D(x)=D_{x}$ gives rise to a smooth distribution on $M$. Again since $h$ is an immersion and since the span of $\xi_{1}$ and $\xi_{2}$ defines an integrable distribution on $\Sigma_{1} \times \Sigma_{2}, D$ is integrable on $M$. Clearly each leaf of $D$ is a compact Riemann surface. This can be seen as follows. Let $g$ be the Riemannian metric on $\Sigma_{1} \times \Sigma_{2}$. Then it is easy to see that each fiber in $\Sigma_{1} \times \Sigma_{2}$ is a totally geodesic submanifold of $\Sigma_{1} \times \Sigma_{2}$ with respect to the metric $g$. Let $M$ have the induced Riemannian metric from $g$. By the definition of $D$, we can readily see that $D$ is a totally geodesic distribution on $M$ with respect to the induced metric. Since $M$ is complete with respect to the metric, each leaf must be complete with respect to the induced metric, too. For the details, see Abe [1]. Since $M$ is compact, all the leaves are compact, too. Let $L$ be a leaf of the distribution $D$. Then $h$ restricted to $L$ is an immersion and $h(L)$ is contained in a compact fiber $F$ of $\Sigma_{1} \times \Sigma_{2}$. Since $L$ and $F$ have the same dimension, $h \mid L$ is a covering mapping. For this part, see Kobayashi-Nomizu [17, Vol. 1, p. 176]. Now since $F$ is diffeomorphic to the torus $S^{1} \times S^{1}$, we can put a flat Riemannian metric on $F$. Since $h \mid L$ is a covering projection, we can lift the metric on $F$ to $L$, and $L$ becomes a flat manifold with respect to the metric. Then by Gauss-Bonnet theorem, the genus of $L$ must be 1 ; therefore, $L$ is an elliptic curve. 
Next let $B=M / D$ be the quotient space of $M$ with respect to $D$. Since $h$ is a local diffeomorphism which maps the leaves of $D$ into the fibers of $\Sigma_{1} \times \Sigma_{2}$, the quotient topology of $B$ is locally the same as that of the image of $h(M)$ under $\pi_{1} \times \pi_{2}$. Note here that $\pi_{1} \times \pi_{2}$ being proper and holomorphic implies that $\left(\pi_{1} \times \pi_{2}\right)(h(M))$ is an analytic subvariety of the analytic space (complex space) $\Sigma_{1} \times \Sigma_{2}$. (See [11].) Thus we have naturally induced continuous mapping $\bar{h}: B \rightarrow B_{1} \times B_{2}$ which is a local homeomorphism. Now let $\mathcal{O}\left(B_{1} \times B_{2}\right)$ denote the structure sheaf of the analytic space, then the induced sheaf $\bar{h}^{*} \mathcal{O}\left(B_{1} \times B_{2}\right)$ gives rise to an analytic structure sheaf of $B$ where $\bar{h}^{*}$ is the morphism induced by the mapping $h$. It is also easy to see that $B$ and $B_{1} \times B_{2}$ are locally isomorphic to each other as analytic spaces. To show that $\pi: M \rightarrow B$ is holomorphic, let $\bar{f}$ be a local section of $\bar{h}^{*} \mathscr{O}\left(B_{1} \times B_{2}\right)$. Then there is a local section $\bar{g}$ of $\mathcal{O}\left(B_{1} \times B_{2}\right)$ such that $\bar{h}^{*}(\bar{g})=\bar{f}$, i.e., $\bar{f}=\bar{g} \circ \bar{h}$, where 。 denotes the composition of $\bar{g}$ and $\bar{h}$. Therefore, $\pi^{*}(\bar{f})=\pi^{*} \bar{h}^{*}(\bar{g})=\bar{g} \circ \bar{h} \circ \pi$. Since the above diagram clearly commutes by the definition of $\bar{h}, \bar{g} \circ \bar{h} \circ \pi=$ $\bar{g} \circ\left(\pi_{1} \times \pi_{2}\right) \circ h$. Since $\left(\pi_{1} \times \pi_{2}\right)$ and $h$ are holomorphic, the composition of them is holomorphic. This means that $\pi^{*}(\bar{f})=\pi^{*}\left(\bar{h}^{*}(\bar{g})\right)=\bar{g} \circ \bar{h} \circ \pi=$ $\bar{g} \circ\left(\pi_{1} \times \pi_{2}\right) \circ h$ is in the structure sheaf of $M$, showing that $\pi$ is holomorphic in the sense of analytic space. This completes the proof of Theorem 5 .

q.e.d.

The following lemma may be convenient to clarify the situation.

LEMMA 5. Let $\left(\Sigma_{1} \times \Sigma_{2}, \pi_{1} \times \pi_{2}, B_{1} \times B_{2}\right)$ be the analytic fibration as before, and let $Y$ be any analytic space. Let $h$ be a holomorphic mapping from $\Sigma_{1} \times \Sigma_{2}$ into $Y$ such that each fiber of $\Sigma_{1} \times \Sigma_{2}$ is mapped into a point. Then $h$ induces a holomorphic mapping $\bar{h}$ from $B_{1} \times B_{2}$ into $Y$ such that the following diagram commutes.

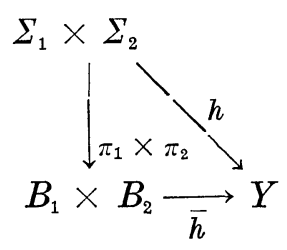

Proof. Let us denote by $\Sigma_{1 \times \Sigma_{2}} \mathcal{O},_{B_{1} \times B_{2}} \mathcal{O}$ and ${ }_{Y} \mathcal{O}$ the structure sheaves of $\Sigma_{1} \times \Sigma_{2}, B_{1} \times B_{2}$ and $Y$, respectively. It is clear that $\bar{h}$ is naturally defined as a continuous mapping in such a way that the above diagram commutes. Therefore, it is sufficient to show that $\bar{h}$ is holomorphic. Here recall that $B_{1} \times B_{2}$ has the natural Cartesian product structure as its complex structure. As is observed in the previous section, the complex 
structure of $B_{i}(i=1,2)$ is the quotient structure of the Seifert fibration $\left(H^{i}, \pi_{i}, H^{i} / T=B_{i}\right)$. We wish to show that for any $\left(x_{1}, x_{2}\right) \in B_{1} \times B_{2}$ and $y \in Y$ such that $\bar{h}\left(x_{1}, x_{2}\right)=y, \bar{h}^{*}\left({ }_{Y} \mathcal{O}_{y}\right) \subset_{B_{1} \times B_{2}} \mathcal{O}_{\left(x_{1}, x_{2}\right)}$. Let $\left(U, \phi, W, C^{k}\right)$ be a local coordinate system of $Y$ at $y$, and let $\left(U_{i}, \phi_{i}, W_{i}, C^{k i}\right)$ be a local coordinate system of $B_{i}$ at $x_{i}(i=1,2)$. This means that for instance, $U$ is a neighborhood of $y$, and $W$ is a local analytic subvariety of $C^{k}$ such that there exists a homeomorphism $\phi$ between $U$ and $W$ such that $\phi^{*}$ gives an isomorphism between the structure sheaves ${ }_{U} \mathcal{O}$ and ${ }_{w} \mathcal{O}$ of $U$ and $W$, respectively. By the definition of Cartesian product of two subvarieties [11], the local coordinate system of $B_{1} \times B_{2}$ at $\left(x_{1}, x_{2}\right)$ is given by the product $\left(U_{1} \times U_{2}, \phi_{1} \times \phi_{2}, W_{1} \times W_{2}, C^{k_{1}+k_{2}}=\boldsymbol{C}^{k_{1}} \times \boldsymbol{C}^{k_{2}}\right)$. Thus it is sufficient to show that for any local section $f$ of ${ }_{U} \mathcal{O}, f \circ \bar{h}$ is a local section of ${ }_{U_{1} \times U_{2}} \mathcal{O}$. Equivalently, $\bar{f} \circ \phi \circ \bar{h} \circ\left(\phi_{1} \times \phi_{2}\right)^{-1}$ is a local section of ${ }_{W_{1} \times W_{2}} \mathcal{O}$, where $\bar{f}$ is a holomorphic function on $W$ such that $\bar{f} \circ \phi=f$. Now restrict $f \circ \bar{h}$ to $U_{i}(i=1,2)$, and denote it by $\psi_{i}(i=1,2)$. Since $B_{i}$ has the quotient complex structure of $H_{i} / T_{i}$, it is sufficient to show that $\psi_{i} \circ \pi_{i}$ is holomorphic in order to show that $\psi_{i}$ is holomorphic. It is quite easy to show that $H_{i}$ is diffeomorphic to $S^{1} \times \Sigma_{i}$, or more precisely via this diffeomorphism the complex structure of $S^{1} \times \Sigma_{i}$ is identified with that of $H_{i}$. In other words, $H_{i}$ is biholomorphic to $S^{1} \times \Sigma_{i}$. Note here that the complex structure $J_{i}$ restricted to $\Theta_{i}$ is always the same as the induced complex structure from the ambient complex Euclidean space as was observed earlier. Let $(a, b) \in \Sigma_{1} \times \Sigma_{2}$ be a point such that $\pi_{1} \times \pi_{2}(a, b)=\left(x_{1}, x_{2}\right)$, and let $\overline{S^{1}}$ be the orbit of the $S^{1}$-action on $\Sigma_{2}$ through $b \in \Sigma_{2}$. Then $\Sigma_{1} \times \overline{S^{1}}$ is a naturally imbedded complex submanifold of $\Sigma_{1} \times \Sigma_{2}$. Since this $S^{1-}$ action has a finite cyclic group as the isotropy group, we have the natural immersion $j$ from $\Sigma_{1} \times S^{1}$ onto $\Sigma_{1} \times \overline{S^{1}} \subset \Sigma_{1} \times \Sigma_{2}$ in such a way that $j(m, n)=(m, t(n))$, where $t: n \rightarrow t(n)$ is the $S^{1}$-action for any $(m, n) \epsilon$ $\Sigma_{1} \times S^{1}$. By this immersion the complex structure $J_{1}$ restricted to $\Theta_{1}$ is mapped precisely onto the complex structure $J$ of $\Sigma_{1} \times \Sigma_{2}$ restricted to $\Theta_{1}$. (Note here this immersion may not be holomorphic.) It is also clear that $h \circ j=\bar{h} \circ\left(\pi_{1} \times \pi_{2}\right) \circ j=\bar{h} \circ \pi_{1}$ as mappings on any sufficiently small open subsets of $\pi_{1}^{-1}\left(U_{1}\right)$. Let $v$ be a point in $\pi_{1}^{-1}\left(x_{1}\right)$, and let $\left(V, \gamma, V^{\prime}, C^{l}\right)$ be a local coordinate system of $\Sigma_{1} \times S^{1}$ about $v$ such that $V$ is so small that the above equalities hold. Then $\bar{f} \circ \phi \circ \bar{h} \circ \pi_{1} \circ \gamma^{-1}=\bar{f} \circ \phi \circ h \circ j \circ \gamma^{-1}$ is a smooth function in the usual sense, since $\bar{f} \circ \phi \circ h$ is a holomorphic function in the usual sense in the open subset $j(V)=j \circ \gamma^{-1}\left(V^{\prime}\right)$ of $\Sigma_{1} \times \Sigma_{2}$, and since $j \circ \gamma^{-1}$ is a smooth mapping from $V^{\prime}$ onto $j(V)$. Since $\Sigma_{1} \times S^{1}$ is a complex manifold, the coordinate system $\left(V, \gamma, V^{\prime}, \boldsymbol{C}^{l}\right)$ is the usual local coordinate system of $\Sigma_{1} \times S^{1}$ about $v$. Thus, in order to show that $\bar{f} \circ \phi \circ h \circ j \circ \gamma^{-1}$ is 
holomorphic, it is sufficient to show that the Jacobian map $\left(\bar{f} \circ \phi \circ h \circ j \circ \gamma^{-1}\right)_{*}$ of $\bar{f} \circ \phi \circ h \circ j \circ \gamma^{-1}$ is a complex linear mapping in the sense that it commutes with the complex structures on $\Sigma_{1} \times S^{1}$ and $C$. This can be seen easily as follows. First of all, we know that the Jacobian map $(\bar{f} \circ \phi \circ h)_{*}$ of $\bar{f} \circ \phi \circ h$ is a complex linear map, since $\bar{f} \circ \phi \circ h$ is holomorphic in $j(V)$ : Next let $\left\{e_{1}, \cdots, e_{2 k}\right\}$ be a $2 k$-frame on $V^{\prime}$ such that $\gamma_{*}^{-1}\left(e_{1}\right), \gamma_{*}^{-1}\left(e_{2}\right)$ span the tangent space to the fibers on $\Sigma_{1} \times S^{1}$ and the rest span $\Theta_{1}$. By the definition of $j$, the Jacobian map $\left(j \circ \gamma^{-1}\right)_{*}$ of $j \circ \gamma^{-1} \operatorname{maps}\left\{e_{1}, e_{2}\right\}$ into the tangent space of the fibers. Again by the definition of $j$, the Jacobian map of $j$ restricted to $\Theta_{1}$ commutes with the complex structures $J_{1}$ restricted to $\Theta_{1}$ and $J$ restricted to $\Theta_{1}$. Note that $J_{1} \mid \Theta_{1}$ and $J \mid \Theta_{1}$ are nothing but the same structure by the definition of the complex structure of $\Sigma_{1} \times \Sigma_{2}$. Since $\bar{f} \circ \phi \circ h$ maps a fiber into a point, the Jacobian map $\left(\bar{f} \circ \phi \circ h \circ j \circ \gamma^{-1}\right)_{*}$ of $\bar{f} \circ \phi \circ h \circ j \circ \gamma^{-1}$ maps the span of $e_{1}$ and $e_{2}$ into the zero vector. For any $e_{i}(2 \leqq i \leqq 2 k),\left(\bar{f} \circ \phi \circ h \circ j \circ \gamma^{-1}\right) J_{1} e_{i}=(\bar{f} \circ \phi \circ h)_{*} J\left(j \circ \gamma^{-1}\right)_{*} e_{i}=$ $J_{0}\left(\bar{f} \circ \phi \circ h \circ j \circ \gamma^{-1}\right) e_{i}$, where $J_{0}$ is the standard complex structure of $C$. Thus $\left(\bar{f} \circ \phi \circ h \circ j \circ \gamma^{-1}\right)$ is a complex linear mapping. Thus we have shown that $\psi_{1} \circ \pi_{1}$ is holomorphic, i.e., $\psi_{1}$ is holomorphic. Applying the same argument, we can show that $\psi_{2}$ is holomorphic. This tells us for any $\left(x_{1}, x_{2}\right) \in B_{1} \times$ $B_{2}, \bar{h}$ restricted to $\left(x_{1}, B_{2}\right)$ and $\left(B_{1}, x_{2}\right)$ are holomorphic. Then by the generalized version of Hartogs' theorem [9], $\bar{h}$ is holomorphic in $B_{1} \times B_{2}$.

q.e.d.

CoRollary 4. Let $\left(\Sigma_{1} \times \Sigma_{2}, \pi_{1} \times \pi_{2}, B_{1} \times B_{2}\right)$ and $\left(\Sigma_{3} \times \Sigma_{4}, \pi_{3} \times \pi_{4}, B_{3} \times B_{4}\right)$ be two fibrations as given in Theorem 3. Let $h: \Sigma_{1} \times \Sigma_{2} \rightarrow \Sigma_{3} \times \Sigma_{4}$ be any holomorphic mapping from $\Sigma_{1} \times \Sigma_{2}$ into $\Sigma_{3} \times \Sigma_{4}$. Then $h$ maps any fiber of $\Sigma_{1} \times \Sigma_{2}$ into a fiber of $\Sigma_{3} \times \Sigma_{4}$; therefore, $h$ induces a holomorphic mapping $\bar{h}$ from $B_{1} \times B_{2}$ into $B_{3} \times B_{4}$ such that following diagram commutes.

$$
\begin{aligned}
& \Sigma_{1} \times \Sigma_{2} \stackrel{h}{\longrightarrow} \Sigma_{3} \times \Sigma_{4} \\
& \downarrow \pi_{1} \times \pi_{2} \quad \downarrow \pi_{3} \times \pi_{4} \\
& B_{1} \times B_{2} \underset{\bar{h}}{\longrightarrow} B_{3} \times B_{4} \text {. }
\end{aligned}
$$

In particular, if $h$ is one to one, then each fiber of $\Sigma_{1} \times \Sigma_{2}$ is mapped biholomorphically onto a fiber of $\Sigma_{3} \times \Sigma_{4}$.

Proof. It is sufficient to point out that each fiber of $\Sigma_{1} \times \Sigma_{2}$ is mapped onto a subvariety of complex dimension 1; therefore, it must be contained in a torus by Theorem 5. If $h$ is one to one, $h$ restricted to a fiber of $\Sigma_{1} \times \Sigma_{2}$ must map the fiber onto a fiber of $\Sigma_{3} \times \Sigma_{4}$; therefore, it is biholomorphic on the fiber.

q.e.d. 
The following corollaries are essentially due to Calabi-Eckmann [6].

Corollary 5. Let $\left(\Sigma_{1} \times \Sigma_{2}, \pi_{1} \times \pi_{2}, B_{1} \times B_{2}\right)$ be a fibration as before. Let $W$ be a subvariety of $\Sigma_{1} \times \Sigma_{2}\left(W\right.$ can be $\Sigma_{1} \times \Sigma_{2}$ itself $)$. Then there exists a 2-cycle in $H^{2}(W, \boldsymbol{R})$ which is represented by a compact complex analytic submanifold of $W$. In particular, if $H^{2}(W, \boldsymbol{R})=0, W$ cannot be Kählerian.

Proof. Take the fibers of the induced fibration of $W$. These are topologically tori, thus represent 2-cycles. If $H^{2}(W, \boldsymbol{R})=0$, these cycles are homologous to 0 . Since no 2-cycle represented by a compact complex submanifold in a Kählerian manifold can be homologous to $0, W$ cannot be Kählerian.

q.e.d.

This also shows that many $\Sigma_{1} \times \Sigma_{2}$ cannot be Kählerian since many of $\Sigma_{1}$ and $\Sigma_{2}$ are highly connected.

Corollary 7. Let $\left(\Sigma_{1} \times \Sigma_{2}, \pi_{1} \times \pi_{2}, B_{1} \times B_{2}\right)$ be a fibration as above. Let $h: \Sigma_{1} \times \Sigma_{2} \rightarrow S^{2}$ be any holomorphic mapping into the Riemannian sphere. This means that $h$ is a meromorphic function on $\Sigma_{1} \times \Sigma_{2}$. Then $h$ is constant on each fiber; therefore, it induces a holomorphic mapping $\bar{h}$ from $B_{1} \times B_{2}$ into $S^{2}$ such that the following diagram commutes.

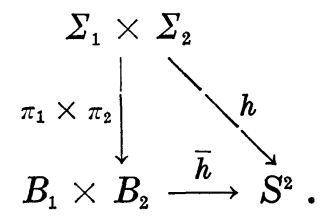

Indeed, the same result holds for any holomorphic mapping $h: \Sigma_{1} \times \Sigma_{2} \rightarrow X$, where $X$ is an analytic space.

Proof. Let $y$ be a point in $S^{2}$. Then $h^{-1}(y)$ is a closed analytic subvariety of $\Sigma_{1} \times \Sigma_{2}$. By the previous theorem, $h^{-1}(y)$ is fibered by elliptic curves induced from the fibration on $\Sigma_{1} \times \Sigma_{2}$. This means that if $x$ is a point such that $h(x)=y$, then the fiber that contains $x$ is entirely contained in $h^{-1}(y)$. Therefore, $h$ restricted to the fiber is constant. By Lemma 5 , there is a unique holomorphic mapping $\bar{h}$ which satisfies the above condition.

q.e.d.

Corollary 8. Let $\left(\Sigma_{1} \times \Sigma_{2}, \pi_{1} \times \pi_{2}, B_{1} \times B_{2}\right)$ be given as before, and let $\left\{a_{1}, \cdots, a_{n}\right\}$ be a finite subset of $\Sigma_{1} \times \Sigma_{2}$. Then $\Sigma_{1} \times \Sigma_{2}-\left\{a_{1}, \cdots, a_{n}\right\}$ is an open complex manifold which contains 2-cycles represented by elliptic curves. Thus most of $\Sigma_{1} \times \Sigma_{2}-\left\{a_{1}, \cdots, a_{n}\right\}$ cannot admit $a$ Kählerian structure. Now let $h$ be a holomorphic function defined on 
$\Sigma_{1} \times \Sigma_{2}-\left\{a_{1}, \cdots, a_{n}\right\}$. Then $h$ is constant.

Proof. Notice that $\Sigma_{1} \times \Sigma_{2}-\left\{a_{1}, \cdots, a_{n}\right\}$ are fibered by elliptic curves and possibly elliptic curves with finite points removed. Those elliptic curves are dense everywhere on the set, and they represent 2cycles. Next by using the homology exact sequence of the pair $\left(\Sigma_{1} \times \Sigma_{2}\right.$, $\left.\Sigma_{1} \times \Sigma_{2}-\left\{a_{1}, \cdots, a_{n}\right\}\right)$ and the excision theorem, we can readily show that $H_{2}\left(\Sigma_{1} \times \Sigma_{2} ; \boldsymbol{R}\right)$ is isomorphic to $H_{2}\left(\Sigma_{1} \times \Sigma_{2}-\left\{a_{1}, \cdots, a_{n}\right\} ; \boldsymbol{R}\right)$. Since most of $\Sigma_{1} \times \Sigma_{2}$ are more than 2-connected as mentioned before, $H_{2}\left(\Sigma_{1} \times\right.$ $\left.\Sigma_{2} ; \boldsymbol{R}\right) \cong H_{2}\left(\Sigma_{1} \times \Sigma_{2}-\left\{a_{1}, \cdots, a_{n}\right\} ; \boldsymbol{R}\right) \cong H_{2}\left(\Sigma_{1} \times \Sigma_{2} ; \boldsymbol{Z}\right) \boldsymbol{\Theta}_{\boldsymbol{Z}} \boldsymbol{R} \cong \pi_{2}\left(\Sigma_{1} \times \Sigma_{2}\right) \times$ $\otimes_{Z} R=0$. The last equality comes from Hurewicz isomorphism theorem. Finally, $h$ restricted to any elliptic fiber is constant by the maximum principle. Since such elliptic fibers are dense in $\Sigma_{1} \times \Sigma_{2}, f$ restricted to any type of fiber must be constant along the fiber. Thus by the similar argument to Lemma $1, h$ induces a holomorphic mapping $\bar{h}$ from $B_{1} \times B_{2}$ into $\boldsymbol{C}$ such that the following diagram commutes.

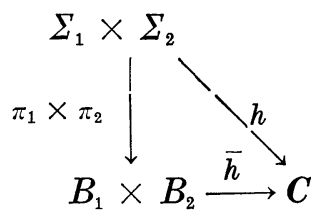

Since $B_{1} \times B_{2}$ are compact and connected, $\bar{h}$ is constant by the maximum principle for analytic spaces. For the details, see Gunning-Rossi [11].

Corollary 9. Let $\left(\Sigma_{1}, \pi_{1}, B_{1}\right)$ and $\left(\Sigma_{2}, \pi_{2}, B_{2}\right)$ be the triples such that $\Sigma_{1}$ and $\Sigma_{2}$ are Brieskorn spheres, and let $\left(\Sigma_{1} \times \Sigma_{2}, \pi_{1} \times \pi_{2}, B_{1} \times B_{2}\right)$ be the fibration. Denote by $\Sigma_{1}-\{a\}$ (or $\Sigma_{2}-\{b\}$ ) the open subset of $\Sigma_{1}$ (or $\Sigma_{2}$ ) obtained by removing a point $\{a\}$ (or $\left.\{b\}\right)$ from $\Sigma_{1}$ (or $\left.\Sigma_{2}\right)$. It is then easy to see that $\left(\Sigma_{1}-\{a\}\right) \times\left(\Sigma_{2}-\{b\}\right)$ is homeomorphic to a complex Euclidean space. It contains infinitely many 2-cycles homologous to zero. It neither can admit a Kählerian structure, nor be covered by a single complex coordinate system, nor admit any non-constant holomorphic function.

Proof. As before, notice that $\left(\Sigma_{1}-\{a\}\right) \times\left(\Sigma_{2}-\{b\}\right)$ is fibered by elliptic curves, $\boldsymbol{C}$ and cylinders $S^{1} \times \boldsymbol{R}$. Of course, each of these fibers is contained in a fiber of $\Sigma_{1} \times \Sigma_{2}$. Again the elliptic fibers are everywhere dense in the set. Next it is easy to see that this complex manifold is homeomorphic to some complex Euclidean space. (Actually, it is diffeomorphic to a complex Eucidean space, because the latter admits the unique differentiable structure.) Therefore, it is contractible. This implies 
$H^{2}\left(\left(\Sigma_{1}-\{a\}\right) \times\left(\Sigma_{2}-\{b\}\right) ; \boldsymbol{R}\right)=0$. So it cannot be Kählerian. The last assertion can be shown in the same way as before. Now suppose that $\left(\Sigma_{1}-\{a\} \times\left(\Sigma_{2}-\{b\}\right)\right.$ admits a single complex coordinate system. Then every coordinate function gives rise to a holomorphic function on it; therefore, constant. This is a contradiction. This completes the proof. q.e.d.

Let us denote by $M$ an analytic space, and by $\operatorname{Aut}(M)$ the set of all the automorphisms of $M$, i.e., the set of all biholomorphic mappings of $M$ onto itself. It is well known that if $M$ is a compact complex manifold, Aut $M$ is a complex Lie group and its Lie algebra aut $M$ is identified with the set of all the holomorphic vector fields on $M$. To be more precise, let $X$ be a vector field on $M . \quad X$ generates a local 1parameter group of local transformations. Denote by $J$ the complex structure on $M$. A vector field $X$ on $M$ is said to be an infinitesimal automorphism of $J$ on $M$ if $L_{X} J=0$, where $L_{X}$ is the Lie differentiation in $X$ direction. This condition is the same as $[X, J Y]=J[X, Y]$ for all vector fields $Y$ on $M$. It is easy to see that this condition is also equivalent to the one that the local transformations generated by $X$ are holomorphic. Since $M$ is compact, all the vector fields generate a global 1-parameter group of holomorphic transformations (or automorphisms). Thus via the exponential map, aut $M$ can be identified with the set of all the infinitesimal automorphisms of $J$ on $M$, each of which is identified with a holomorphic vector field $Z$ on $M$ by $X \mapsto(X-i J X) / 2=Z$. For more details, see [16] or [17].

Now we state a result related to the last corollary.

CoRollary 10. Let $\left(\Sigma_{1} \times \Sigma_{2}, \pi_{1} \times \pi_{2}, B_{1} \times B_{2}\right)$ be an analytic fibration. Then there exists a homomorphism $h$ : Aut $\left(\Sigma_{1} \times \Sigma_{2}\right) \rightarrow$ Aut $\left(B_{1} \times B_{2}\right)$. The set of infinitesimal automorphisms induced by the kernel of $h$, say $\operatorname{ker} h$, is isomorphic to $\boldsymbol{C}$. Here $\boldsymbol{C}$ is considered to be the vector space over $\boldsymbol{C}$.

Proof. Let $\phi$ be an element of Aut $\left(\Sigma_{1} \times \Sigma_{2}\right)$. By Theorem 4 each fiber is mapped onto a fiber biholomorphically. By Lemma $5 \phi$ induces a holomorphic mapping $\bar{\phi}: B_{1} \times B_{2} \rightarrow B_{1} \times B_{2}$ such thah $\pi_{1} \times \pi_{2} \circ \phi=\bar{\phi} \circ \pi_{1} \times \pi_{2}$. Clearly $\bar{\phi}$ belongs to Aut $\left(B_{1} \times B_{2}\right)$. Define the homomorphism $h$ : Aut $\left(\Sigma_{1} \times \Sigma_{2}\right) \rightarrow$ Aut $\left(B_{1} \times B_{2}\right)$ by $h(\phi)=\bar{\phi}$. It is easy to see that $h$ is a homomorphism, and the proof is left to the reader. Next since an element $\phi$ of Ker $h$ leaves all the fibers fixed, $\phi$ induces an automorphism on each fiber. Let $L$ denote a fiber. Then $\phi \mid L$ is an automorphism of $L$. Since $L$ is an elliptic curve, the associated infinitesimal automorphism to $\phi \mid L$ on $L$ is a constant vector field. In other words, it is a vector field represented 
by a constant vector in the universal covering space $C$. Going back to $\phi$ itself, let $X$ be the infinitesimal automorphism associated to $\phi$ on $\Sigma_{1} \times$ $\Sigma_{2}$. Note that we can identify $\phi$ and $X$, since $\Sigma_{1} \times \Sigma_{2}$ is compact. Then $X$ satisfies the identity $[X, J Y]=J[X, Y]$ for any vector field $Y$ on $\Sigma_{1} \times$ $\Sigma_{2}$. Let $E$ be the subbundle of $T\left(\Sigma_{1} \times \Sigma_{2}\right)$ generated by $\xi_{1}$ and $\xi_{2}$. Then by the above observation $X$ is a smooth section of $E$, and $(X-i J X) / 2$ is a holomorphic vector field on $\Sigma_{1} \times \Sigma_{2}$ which is constant on each fiber. Next we show that $E$ is a trivial bundle generated by two infinitesimal automorphisms. Clearly $\xi_{i}(i=1,2)$ generates $E$. Then by the same argument as in the proof of Lemma 4, Part I, we can show that $L_{\xi_{i}} J=0$ $(i=1,2)$. Thus $\left(\xi_{j}-i J \xi_{j}\right) / 2(j=1,2)$ are holomorphic vector fields, and these are linearly dependent over $C$. If a vector field $X$ is an infinitesimal automorphism of $J$ and a linear combination of $\xi_{1}$ and $\xi_{2}$, then $(X-i J X) / 2$ is a holomorphic vector field and linearly dependent of $\left(\xi_{1}-i J \xi_{1}\right) / 2$, over $\boldsymbol{C}$. Thus, $(X-i J X) / 2$ is a holomorphic section of the trivial holomorphic bundle $\left(\Sigma_{1} \times \Sigma_{2}\right) \times C$ generated by $\left(\xi_{1}-i J \xi_{1}\right) / 2$. Let $P$ be the natural projection of $\left(\Sigma_{1} \times \Sigma_{2}\right) \times C$ onto $C$. Then $P$ is a holomorphic mapping; and therefore, $P \circ Z$ is a holomorphic function in $\Sigma_{1} \times \Sigma_{2}$ for any holomorphic vector field $Z$. Since $\Sigma_{1} \times \Sigma_{2}$ is compact, $P \circ Z$ must be a constant function. This gives us the identification of $Z$ with a complex number which is the value of $P \circ Z$. Conversely, any such a constant gives rise to a holomorphic vector field, and consequently an infinitesimal automorphism in the kernel of $h$. Thus, $\operatorname{ker} h$ is isomorphic to $C$ over $C$. q.e.d.

As a special case we have,

CoRollary 11. Let $\left(\Sigma_{1} \times \Sigma_{2}, \pi_{1} \times \pi_{2}, B_{1} \times B_{2}\right)$ be a fibration as before. If Aut $\left(B_{1} \times B_{2}\right)$ is a finite group, the Lie algebra of Aut $\left(\Sigma_{1} \times \Sigma_{2}\right)$ is equal to the Lie algebra of $\operatorname{Ker} h$, where $h$ : Aut $\left(\Sigma_{1} \times \Sigma_{2}\right) \rightarrow \operatorname{Aut}\left(B_{1} \times B_{2}\right)$ is the above homomorphism. Thus, Aut $\left(\Sigma_{1} \times \Sigma_{2}\right)=C$.

REMARK. It is of some interest to notice that there are quite a few complex manifolds which have a finite automorphism group. As for the base space $B_{1} \times B_{2}$, some sufficient conditions for $B_{i}(i=1,2)$ to be a complex manifold have been obtained in the case of Examples 1, 2 and 3; see [4], [23], [24]. Thus it makes sense to speak of some sufficient conditions for a compact complex manifold to have a finite automorphism group. The following are those conditions. a) $B_{1} \times B_{2}$ is a complex manifold and has negative first Chern class. b) $B_{1} \times B_{2}$ is hyperbolic manifold in the sense of Kobayashi. c) $B_{1} \times B_{2}$ is a projective algebraic manifold of general type and finally, d) $B_{1} \times B_{2}$ is a compact Riemann surface of genus $\geqq 2$. For the details, see Kobayashi [16]. In particular, 
d) is of greatest interest because of the following reasons. As we pointed out earlier, our induced $S^{1}$-action on $\Sigma$ gives only principal orbits and exceptional orbits without fixed point if dimension of $\Sigma=3$; therefore, the orbit space $\Sigma / S^{1}=B$ is always a manifold. In fact, it has been pointed out [23] [24] that $B$ is a topological manifold if and only if $B$ is a complex manifold with the induced complex structure and the quotient map is holomorphic. Combining these, we see that if $\operatorname{dim} \Sigma=3$, the fibration $\left(S^{1} \times \Sigma, \pi, B\right)$ is an analytic fibration with a compact Riemann surface $B$. For instance, if $\Sigma$ is an original Brieskorn 3-manifold, Neumann's characterization gives us that there are plenty of compact Riemann surfaces of genus $\geqq 2$ which arise as an orbit space $B=\Sigma / S^{1}$. As for the Chern classes of $\Sigma_{1} \times \Sigma_{2}$, the highest Chern class of $\Sigma_{1} \times \Sigma_{2}$ always vanishes. This can be seen as follows. The tangent bundle $T\left(\Sigma_{1} \times \Sigma_{2}\right)$ can be expressed $\operatorname{Sp}\left(\xi_{1}, \xi_{2}\right) \oplus\left(\Theta_{1} \oplus \Theta_{2}\right)$ as a complex vector bundle, where $\operatorname{Sp}\left(\xi_{1}, \xi_{2}\right)$ denotes the span of $\xi_{1}$ and $\xi_{2}$. Sp $\left(\xi_{1}, \xi_{2}\right)$ is a trivial complex line bundle over $\Sigma_{1} \times \Sigma_{2}$ and has the trivial Chern classes. By the Whitney sum axiom, we have the desired result. In particular we have,

CoRollary 12. Let $\Sigma_{1} \times \Sigma_{2}$ be a complex manifold given as before. Then its Chern numbers vanish.

Proof. By the theorem of Bott [3], we know that if $\Sigma_{1} \times \Sigma_{2}$ admit a non-vanishing holomorphic vector field, then all the characteristic numbers (complex case) vanish. Since $\xi_{i}(i=1,2)$ is such a holomorphic vector field, in particular, the Chern numbers vanish.

q.e.d.

We have seen that $\Sigma_{1} \times \Sigma_{2}$ as a differentiable manifold can admit a infinitely many seemingly different complex structures as described in Theorem 1. We have also discussed some properties of these complex manifolds. In what follows, we attempt to distinguish these complex structures. Perhaps, it should be mentioned here that there are two generally accepted criteria to classify complex structures on a complex manifold. One is the classification with respect to biholomorphic mappings, and the other is with respect to the notion of complex deformation in the sense of Kodaira-Spencer. We use the former criterion. Note also that Morita [20] recently obtained a classification of complex structures on $S^{1} \times \Sigma^{2 n-1}$ up to the homotopy of the underlying almost complex structures, where $\Sigma^{2 n-1}$ is a Brieskorn homotopy sphere of dimension $2 n-1$. Going back to the first case, let $M$ and $N$ be two complex manifolds. We say that $M$ and $N$ are equivalent as complex manifolds if there exists a biholomorphic map from $M$ onto $N$.

Let $\Sigma_{1} \times \Sigma_{2}$ be the complex manifold and let $\Sigma_{i}(i=1,2)$ have the 
$S^{1}$-action as before. Introduce a smooth torus $T=S^{1} \times S^{1}$-action on $\Sigma_{1} \times \Sigma_{2}$ as follows. Let $(s, t) \in T=S^{1} \times S^{1}$ be an element of $T$, and let $(x, y) \in \Sigma_{1} \times \Sigma_{2}$ be a point of $\Sigma_{1} \times \Sigma_{2}$. Define the $T$-action on $\Sigma_{1} \times \Sigma_{2}$ by $(s, t)(x, y)=(s x, t y)$, where $s x$ and $t y$ denote the $S^{1}$-actions on $\Sigma_{1}$ and $\Sigma_{2}$, respectively. Let $g$ be the Riemannian metric on $\Sigma_{1} \times \Sigma_{2}$ induced naturally from the associated Riemannian structures with $\eta_{1}$ and $\eta_{2}$ on $\Sigma_{1}$ and $\Sigma_{2}$, respectively. Then the torus action on $\Sigma_{1} \times \Sigma_{2}$ can be considered as an isometric action with respect to $g$. Now let $\left[\boldsymbol{Z}_{p}, \sigma_{p_{1}} \oplus \cdots \oplus \sigma_{p_{m}} \oplus \sigma_{p_{0}}\right]$ and let $\left[\boldsymbol{Z}_{q}, \sigma_{q_{1}} \oplus \cdots \oplus \sigma_{q_{n}} \oplus \sigma_{q_{0}}\right]$ be the slice types of $S^{1}$-actions on $\Sigma_{1}$ and $\Sigma_{2}$ at $x$ and $y$, respectively. Here

$$
\sigma_{p_{i}}=\left(\begin{array}{rr}
\cos 2 \pi p_{i} t & -\sin 2 \pi p_{i} t \\
\sin 2 \pi p_{i} t & \cos 2 \pi p_{i} t
\end{array}\right) \quad(0 \leqq i \leqq m)
$$

as the representation of $\boldsymbol{C}=\boldsymbol{R}^{2}$ and $\sigma_{p_{0}}$ is the identity representation of a linear subspace. $\sigma_{q_{j}}(j=1, \cdots, n)$ and $\sigma_{q_{0}}$ should be interpreted similarly. Now it is easy to show that the isotropy group of the $T$-action at $(x, y)$ is precisely $\boldsymbol{Z}_{p} \times \boldsymbol{Z}_{q} \subset S^{1} \times S^{1}$. From the above observations, the slice representation of $\boldsymbol{Z}_{p} \times \boldsymbol{Z}_{q}$ on the normal space to the orbit through $(x, y)$ with respect to $g$ is given by

$$
\left[\boldsymbol{Z}_{p} \times \boldsymbol{Z}_{q} ; \bar{\sigma}_{p_{1}} \oplus \cdots \oplus \bar{\sigma}_{p_{m}} \oplus \bar{\sigma}_{p_{0}} \oplus \bar{\sigma}_{q_{1}} \cdots \oplus \bar{\sigma}_{q_{n}} \oplus \bar{\sigma}_{q_{0}}\right] .
$$

Here $\bar{\sigma}_{p_{i}}$ is the representation of the torus $T=S^{1} \times S^{1}$ given by $\bar{\sigma}_{p_{i}}(s, t)=$ $\sigma_{p_{i}}(s)(i=1, \cdots, m, 0)$, and $\bar{\sigma}_{q_{i}}$ is given by $\bar{\sigma}_{q_{i}}(s, t)=\sigma_{q_{i}}(t)(i=1, \cdots, n, 0)$. Next let us regard the orbits of the $S^{1}$-action and the $T$-action as the leaves of foliations on $\Sigma_{1}, \Sigma_{2}$ and $\Sigma_{1} \times \Sigma_{2}$. Let $\left(U_{1}, x_{1}, \cdots, x_{k}\right)$ and $\left(U_{2}, y_{1}\right.$, $\cdots, y_{l}$ ) be the cubical Frobenius coordinate systems at $x$ and $y$, respectively, such that the slices for the leaves are given by the first coordinates. By the definition of the $T$-action on $\Sigma_{1} \times \Sigma_{2}$, it is clear that $\left(U_{1} \times U_{2}, x_{1}\right.$, $\left.\cdots, x_{k}, y_{1}, \cdots, y_{l}\right)$ gives us a cubical Frobenius coordinate of foliation on $\Sigma_{1} \times \Sigma_{2}$ at $(x, y)$. In this case the slices for the leaves are given by $x_{1}$ and $y_{1}$ coordinates. Suppose that a leaf $L_{1}$ on $\Sigma_{1}$ passing through a point in $U_{1}$ meets $U_{1}$ in $\alpha$ slices, and suppose that a leaf $L_{2}$ on $\Sigma_{2}$ passing through a point in $U_{2}$ meets $U_{2}$ in $\beta$ slices. Then it is clear that the leaf $L_{1} \times L_{2}$ on $\Sigma_{1} \times \Sigma_{2}$ meets $U_{1} \times U_{2}$ in $\alpha \beta$ slices which are all distinct. Thus we have shown that,

Lemma 6. a) The slice diagram of the above torus action on $\Sigma_{1} \times \Sigma_{2}$ is precisely the set of all the slice types described as above.

b) Let $(x, y)$ be a point in $\Sigma_{1} \times \Sigma_{2}$. Assume that the $S^{1}$-orbits of $\Sigma_{1}$ and $\Sigma_{2}$ through $x$ and $y$, respectively, meet cubical Frobenius coordinate neighborhoods of $\Sigma_{1}$ and $\Sigma_{2}$ in $\alpha$ and $\beta$ different slices. Then the product 
orbit of $\Sigma_{1} \times \Sigma_{2}$ meets the product Frobenius coordinate neighborhood in $\alpha \beta$ different slices.

Let $G$ be a compact Lie group acting on a smooth manifold $M$, and let $\Delta(G, M)$ be its slice diagram. Let $[H, \sigma]$ be the slice type of an orbit. We define the intersection number of $[H, \sigma]$ to be the number of slices given as follows in a sufficiently small cubical Frobenius neighborhood of a point in the orbit. Let $U$ be such a Frobenius coordinate neighborhood. Then take a leaf through $U$ which is at the same time a principal orbit of the $G$-action. The intersection number is defined to be the number of slices in which the leaf intersects $U$. Note here that this number neither depends on the choice of points where $U$ is taken, nor the choice of principal orbits. It also does not depend on the size of $U$ as long as $U$ is taken sufficiently small. Finally, this number is constant in the orbit bundle; however, two different orbit bundles can have the same intersection number. Let us denote by $I(G, M)$ the set of ordered pairs $(l, k)$ of integers $l$ and $k$ such that $l$ runs over all the possible intersection numbers associated with each slice type of $\Delta(G, M)$, and $k$ is the total number of connected components of orbit bundles which are associated with the slice types of intersection number $l$. Then we have,

THEOREM 6. Let $\Sigma_{1} \times \Sigma_{2}$ and $\Sigma_{3} \times \Sigma_{4}$ be two products of generalized Brieskorn manifolds. Assume that there is a diffeomorphism $f: \Sigma_{1} \times \Sigma_{2} \rightarrow$ $\Sigma_{3} \times \Sigma_{4}$. Then $f$ cannot be a biholomorphic mapping between them, unless $I\left(T, \Sigma_{1} \times \Sigma_{2}\right)$ and $I\left(T, \Sigma_{3} \times \Sigma_{4}\right)$ are identically the same.

Proof. Assume that $f$ is biholomorphic. By Theorem 5, each fiber of $\Sigma_{1} \times \Sigma_{2}$ must be mapped onto a fiber of $\Sigma_{3} \times \Sigma_{4}$ biholomorphically. Let $L_{1}$ be a fiber of $\Sigma_{1} \times \Sigma_{2}$ considered as an orbit of the $T$-action, and the slice type of $L_{1}$ has intersection number $l$. If we denote by $L_{2}$ the image fiber of $L_{1}$ under $f$, then the slice type of $L_{2}$ must have the same intersection number $l$. This can be seen easily as follows. Let $U$ be a sufficiently small cubical neighborhood at a point $x$ in $L_{1}$. Since $f$ is a diffeomorphism which preserves the leaves, $f(U)$ is a cubical Frobenius neighborhood at $f(x)$. Let $L$ be a principal orbit passing through $U$. Then $f(L)$ meets $f(U)$ in as many different slices as $L$ meets $U$. Since any principal orbit passing through $f(U)$ meets $f(U)$ at least as many times as $f(L)$ meets $f(U)$, the intersection number of $f\left(L_{1}\right)=L_{2}$ is $\geqq l$. Applying the same argument to $f^{-1}$, we have that the intersection number of $L_{2}=l$, too. Thus $I\left(T, \Sigma_{1} \times \Sigma_{2}\right)$ must be identically the same as $I\left(T, \Sigma_{3} \times \Sigma_{4}\right)$.

q.e.d.

Note here that $f$ may not be an equivariant mapping; therefore, we 
cannot conclude that the slice diagrams are the same. Next we give some concrete examples for Theorem 6 .

CoRollary 13. Let $S^{2 n-1}$ be the $(2 n-1)$-dimensional standard sphere, and let us assume that $S^{2 n-1}$ has the normal contact structures given in Example 7 in Part I. Then $S^{1} \times S^{2 n-1}$ admits infinitely many distinct complex structures. In fact, the complex structures are all distinct.

Proof. Let $S^{1} \times S_{p}^{2 n-1}$ and $S^{1} \times S_{q}^{2 n-1}$ be the complex manifold corresponding to the polynomials $P_{p}(Z)=Z_{0}+Z_{1}+Z_{2}^{p}+\cdots+Z_{n}^{p}$ and $P_{q}(Z)=Z_{0}+Z_{1}+Z_{2}^{q}+\cdots+Z_{n}^{q}$, respectively. Then $I\left(T, S^{1} \times S_{p}^{2 n-1}\right)=$ $\{(p, 1),(1,1)\}$ and $I\left(T, S^{1} \times S_{q}^{2 n-1}\right)=\{(q, 1),(1,1)\}$. Thus if $p \neq q$, they are not the same complex structures.

q.e.d.

It is clear that we can indeed give more precise criterion than that in Theorem 6. For instance, even if $I\left(T, \Sigma_{1} \times \Sigma_{2}\right)$ and $I\left(T, \Sigma_{3} \times \Sigma_{4}\right)$ agree, there cannot exist a biholomorphic mapping between them unless the dimensions of corresponding orbit bundles agree (as submanifolds). From this point of view, we immediately see that the complex structures on $S^{1} \times S^{2 n-1}$ corresponding to the polynomials $P_{i}(Z)=Z_{0}+\cdots+Z_{i}+Z_{i+1}^{p}+$ $\cdots+Z_{n}^{p}(i=1, \cdots, n)$ are all distinct, since the orbit bundle associated with the intersection number $p$ has $2 i-1$ as its real dimension $(i=1, \cdots, n)$. Thus they are distinct complex structures on $S^{1} \times S^{2 n-1}$. In general, the complex structures corresponding to $P_{i, q}(Z)=Z_{0}+\cdots+Z_{i}+Z_{i+1}^{q}+\cdots+Z_{n}^{q}$ $(i=1, \cdots, n, q=1,2, \cdots)$ are all distinct. It is also clear that we can make many more distinct complex structures on $S^{1} \times S^{2 n-1}$.

COROLLARY 14. There are infinitely many Brieskorn exotic spheres whose product with $S^{1}$ admits infinitely many distinct complex structures.

Proof. Use Example 9 in Part I and Theorem 6. q.e.d.

These corollaries are particularly interesting in the following context. As is pointed out earlier, Morita showed that if $n \equiv 1(\bmod 4)$ or $n \equiv 3$ $(\bmod 4), S^{1} \times \Sigma^{2 n-1}(n \geqq 2)$ admits a finite number of complex structures up to almost complex deformation, where $\Sigma^{2 n-1}$ is a Brieskorn sphere. We know practically nothing as to which of the above complex structures are distinct up to deformation.

CoRollary 15. Let $P_{i}(Z)=Z_{0}+Z_{1}^{a_{i}}+Z_{2}^{b_{i}}(i=1,2)$ be as in Theorem 6 in Part I. Then the complex structures on $S^{1} \times S^{3}$ associated with $P_{i}(i=1,2)$ are distinct if the powers of $P_{i}(Z)$ satisfy the same conditions as in Theorem 6 in Part I.

Proof. Under the given conditions, the $I\left(T, S^{1} \times S^{3}\right)$ 's associated with $P_{i}(Z)(i=1,2)$ are different. Now use Theorem 6 . q.e.d. 
Note here that these surfaces (complex surfaces) must be equivalent to one of so called Hopf type surfaces by the result of Kodaira [20]. Finally, let $M$ be a compact complex manifold of dimension $n$, and let $K(M)$ be the set of all meromorphic functions on $M$. It is well known that $K(M)$ is a field and it is finitely generated over $C$. Also its degree of transcendency $d$ is $\leqq n$. Now let $S^{1} \times \Sigma$ be a complex surface given as generalized Brieskorn complex manifold. It is known that if $d=2, S^{1} \times \Sigma$ is projective algebraic; therefore, for most of $\Sigma$ 's, $d=0$ or 1 . It is also known that if $d=1$, there are a compact Riemann surface $N$ and a holomorphic mapping $\phi$ from $M$ into $N$ such that $\phi^{*}: K(N) \rightarrow K(M)$ gives rise to an isomorphism, and such that $\phi^{-1}(x)$ for $x$ except for a finite number of points, is an elliptic curve. These facts and our observations concerning fibration $\left(S^{1} \times \Sigma, \pi, B\right)$ seem to agree. It seems natural to think that even in the general case, $K\left(\Sigma_{1} \times \Sigma_{2}\right)$ has the degree of transcendency which is strictly less than its complex dimension.

\section{BIBLIOGRAPHY}

[1] K. ABE, Characterization of totally geodesic submanifolds in $S^{N}$ and $C P^{N}$ by an inequality, Tôhoku Math. J. 23 (1971), 219-244.

[2] K. ABE, On a generalization of the Hopf fibration, I, Tôhoku Math. J. 29 (1977), 335-374.

[3] R. Bотт, Vector fields and characteristic numbers, Mich. Math. J. 14 (1967), 231-244.

[4] E. BRIESKorn, Beispiele zur Differentialtopologie von Singularitäten, Inventions Math. 2 (1966), 1-14.

[5] E. BRIESKoRn AND A. VAN DE Ven, Some complex structures on products of homotopy spheres, Topology 7 (1968), 389-393.

[6] E. Calabi and B. Eckmann, A class of compact, complex manifolds which are not algebraic, Ann. of Math. 53 (1953), 494-500.

[7] H. Cartan, Quotient d'un espace analytique par un groupe d'automorphismes, Algebraic geometry and topology, Symp. in honor of Lefschetz, Princeton Univ. Press (1957), $90-102$.

[8] B. GIESECKe, Simpliziele Zerlegungen abzählbarer analytischer Räume, Math. Zeit. 83 (1964), 177-213.

[9] H. Grauert and R. Remmert, Komplexe Räume, Math. Ann. 136 (1958), 245-318.

[10] P. Griffiths and J. Adams, Topics in algebraic and analytic geometry, Math. Notes 13, Princeton Univ. Press.

[11] R. C. Gunning and H. Rossi, Analytic functions of several complex variables, Prentice Hall (1965).

[12] H. Holman, Quotientenräume komplexer Mannigfaltigkeiten nach komplex Lieschen Automorphismengruppen, Math. Ann. 139 (1960), 383-402.

[13] H. Hopf, Zur Topologie der komplexen Mannigfaltigkeiten, in Studies and Essays presented to $R$. Courant, New York (1948).

[14] K. JÄNICH, Differenzielbare G-Mannigfaltigkeiten, Lecture Notes in Math. 59, SpringerVerlag (1968).

[15] L. KaUfFmanN, Link manifolds and periodicity, Bull. Amer. Math. Soc. 79 (1973), 570-573. 
[16] S. KoBAYASHI, Transformation groups in differential geometry, Springer-Verlag 70 (1972).

[17] S. KobayasHI AND K. Nomizu, Foundations of differential geometry, Interscience, Vol. I (1963), II (1969).

[18] K. KodaIRA, Complex structures on $S^{1} \times S^{3}$, Proc. Nat. Acad. Sci. USA 55 (1966), 240-243.

[19] A. Morimoto, On normal almost contact structures, J. Math. Soc. Japan 15 (1963), 420436.

[20] S. MorITA, A topological classification of complex structures on $S^{1} \times \Sigma^{2 n-1}$, Topology 14 (1975), 13-22.

[21] D. MUMFoRD, The topology of normal sigularities of an algebraic surface and a criterion for simplicity, Publ. Math. IHES, No. 9 (1961).

[22] A. Newlander and L. Nirenberg, Complex analytic coordinates in almost complex manifolds, Ann. of Math. 65 (1957), 391-404.

[23] W. D. Neumann, $S^{1}$-actions and the $\alpha$-invariant of their involutions, Bonner Mathematische Schriften 44, Bonn (1970).

[24] R. C. RANDELL, Generalized Brieskorn manifolds with $S^{1}$-actions, Thesis, Univ. of Wisconsin (1973).

[25] G. StolzEnBERG, Volumes, Limits and Extensions of Analytic Spaces, Lecture Notes in Math. 19. Springer-Verlag (1966).

[26] H. WhitNeY, Complex analytic variety, Addison-Wesley (1972).

Department of Mathematics

UNIVERSITY OF CONNECTICUT

STORRS, CONNECTICUT 06268

U.S.A. 